\title{
Development, Validation and Application of a Coupled Reduced-order CFD model for Building Control Applications
}

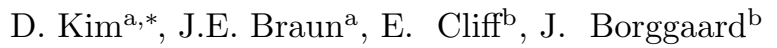 \\ ${ }^{a}$ School of Mechanical Engineering, Purdue University, West Lafayette, IN, USA \\ ${ }^{b}$ Interdisciplinary Center for Applied Mathematics, Virginia Tech, Blacksburg, VA, USA
}

\begin{abstract}
Small-to-moderate sized commercial buildings commonly use rooftop units (RTUs) to provide indoor comfort. These applications are often characterized by significant spatial variations in comfort due to poor thermostat placement and poor coordination of RTUs leading to high energy and demand costs and marginal comfort. Tools are needed that can assess both energy and comfort performance for these types of applications so that the benefits of improved RTU coordination and control can be evaluated in terms of energy and comfort and so that appropriate real-time strategies can be developed. In this paper we discuss a method for generating and coupling tractable reduced order models for the building envelope and for the indoor air dynamics. Overall assessments of the coupled model are performed for a typical sit down restaurant that employs four rooftop units. Measurements are available from the site to validate the models.
\end{abstract}

Keywords: Reduced-order model, CFD, Coupled model, Building model, Building control, RTU

\section{INTRODUCTION}

Building Energy Simulation (BES) tools, such as ENERGYPLUS [1], and TRNSYS [2], are useful in estimating peak cooling and heating demands and annual energy consumption. These tools predict heating/cooling loads and corresponding annual energy consumption required to maintain thermal comfort under a well-mixed assumption for the indoor air. On the other hand, Computational Fluid Dynamics (CFD) tools capture spatial and temporal variations of flow and thermal properties for specified boundary conditions but generally require significant computation time.

\footnotetext{
* Corresponding author

Email address: kim1077@purdue.edu (D. Kim)
}

Preprint submitted to Building and Environment

April 21, 2015 
An integrated BES-CFD model is beneficial when there are significant spatial variations in thermal comfort and air quality inside a building and there is an interest in studying the impact of design and/or control choices on both comfort conditions and energy. Modeling the spatial variation in the indoor environment requires predictions of flow and thermal distributions such as air temperature, relative humidity, mean radiant temperature, air velocity and contaminants, which are difficult to measure in a real application [3]. In a coupled model, the CFD predicts three-dimensional distributions of indoor conditions given boundary conditions, while the BES provides the necessary thermal and flow boundary conditions, e.g. wall surface temperatures or heat flow rates of building enclosures [4]. Sebric et. al. [5] experimentally validated a coupled model for the study of a multi-zone indoor environment. Zhai and Chen [6] and Bartak et. al. [7] also demonstrated the validity of the integrated modeling approach using experiments.

However, for many control applications, it is not generally appropriate to use the coupling approaches that directly utilize commercial CFD tools, due to the high computational demand of CFD. Recall that a building system consists of the building envelope, indoor air, HVAC equipment, and controls. Furthermore to evaluate performance of a control algorithm, e.g. energy consumption and thermal comfort, it is typical to test the controller using the whole building system model for a period of a week/month or sometimes a year. Therefore the direct usage of commercial CFD tools for evaluating the overall performance of system controls would not be practical.. A coupled model that provides an $a p$ proximate solution with reduced computational requirements is needed. There have been some previous studies involving the use of a reduced-order CFD model for control applications. However, they are not generally applicable for analysis of building controls because they do not include an envelope model (See Section 2.1 for more discussion).

A computationally fast and integrated building model is needed for evaluating the benefits of advanced controls for small/medium commercial buildings that utilize rooftop units (RTUs) in open spaces, such as for retail stores, restaurants and factories. A well-mixed assumption is not appropriate for these open-space buildings due to close interactions between the different RTUs and their thermostats, non-uniformly distributed diffusers, and non-uniform heat gains. Unknown but significant inter-zonal air couplings make development of a model very difficult. Tools that employ a fully-mixed assumption for zone air have limited applicability for evaluating control algorithms for RTU coordination because local thermostat temperatures are needed for RTU feedback control and the spatial differences of zone air temperatures cannot be captured with the typical modeling approaches. In the absence of considering spatial temperature variations: 1) all RTUs would be simultaneously activated or be deactivated since their measured temperatures, thermostat signals, are equal for a fully-mixed model; 2) simultaneous on/off actuation of all RTUs would lead to very unrealistic responses of thermostat temperatures to feedback con- 
trol signals compared to reality; 3) the assumption that all RTUs operate with the same on/off cycling could lead to significant errors in prediction of part-load performance and peak electrical demand. These issues are less important for estimation of monthly or annual energy consumption assuming that time-average zone air temperatures track set-points, but are critical for testing a RTU control algorithm in terms of comfort and peak demand. Tools are needed that can assess the performance of RTU coordination and control in terms of both energy and comfort so that appropriate real-time strategies can be developed.

In modeling an open space, one may follow a nodal approach $[8 ; 9 ; 10 ; 11]$ in which the space is divided into several well-mixed subzones and inter-zonal flows are calculated via pressure differences between the subzones. However most of the flow-pressure relationships for large openings are based on a simple orifice model [12] that is not appropriate for air flow couplings in an open space.

The current paper presents a computationally efficient coupled indoor air and envelope modeling approach that is suitable for assessment of advanced control algorithms. The conceptual strategy of the proposed methodology is developed in section 2. In section 3, detailed procedures introduced in section 2 are illustrated for a case study building. Comparisons between the coupled model and measured data for a field site are provided in section 4 .

\section{DEVELOPMENT OF REDUCED ORDER CFD COUPLED MODEL}

\subsection{CFD-BES Coupling Approaches}

There are many different coupling strategies between CFD and BES depending on the usage of a commercial software. For example, one can complete a coupled simulation with an existing CFD tool by adding a new module for solving a set of energy balance equations for wall temperature nodes, to the CFD software. The strategy is called internal coupling [13]. On the other hand, two commercial software packages for CFD and BES can be used for co-simulation, called external coupling. The coupling methods can also be categorized depending on equation sets for the indoor air and wall temperature dynamics. For instance, one could solve partial differential equations of the conversation laws of mass, momentum, energy and/or turbulence, while another could solve an approximated model for the air dynamics to reduce computation time. In this section, literature about the coupling methodologies that were applied to control applications is reviewed and features of our approach compared to the previous research are addressed.

Fan and Ito [14] used software packages, ANSYS-FLUENT and TNRSYS, to design locations of supply/return diffusers by calculating HVAC energy consumption for each candidate layout under a feedback controller. For the co-

simulation, at each BES time step of one hour, a steady CFD calculation was 
carried out and the steady CFD solution was passed to BES. Du, Xu, Jin, and Liu [15] utilized a similar CFD-BES coupling strategy, that directly utilized a full order CFD model, to determine a temperature sensor location. In the paper, a CFD simulation was performed at each BES time step of 5 min. Due to the high computational demand of CFD, the simulation periods were limited to several hours or a day for these coupling approaches that directly utilize commercial CFD tools.

However, for many control applications where many iterations of weekly /monthly simulation may be required, these coupling approaches are not generally appropriate. To tackle the issue, two distinct strategies can be found in literature. Zuo and Chen [16] proposed a numerical algorithm, called Fast Fluid Dyanmics (FFD), that solves Navier-Stokes equation in a numerically efficient manner, for indoor air flow simulation. In their work [17], FFD was coupled to a BES tool, the Modelica Buildings library [18]. Because FFD solves the same equation set as CFD, this approach is very accurate and able to capture non-linear behaviors of indoor air dynamics. Although it is reported that FFD is about 50 times faster than CFD, it is not yet fast enough for some control applications.

On the other hand, Desta et. al. [19; 20] and Brecht et. al. [21] applied system identification methods to extract linear discrete time indoor models that approximate CFD models. The reduced order indoor air models were then used for a model predictive control either to regulate temperature at a position of interest or maintain spatial temperature uniformity. Hiyama et. al. [22] also took a similar approach to tune controller parameters, i.e. proportional and integral gains of a PI controller regulating a zone air temperature with supply air temperature as the manipulated variable. The major advantage of this approach is in a significantly fast computation. In the study of Hiyama, it is reported that CFD analysis for 100 min transient calculation took about 23 hours, while the same analysis with a reduced-order indoor model took only milliseconds. However the approach has limited applications due to the lack of methods interfacing to a envelope model. For this reason, the case studies were limited to air spaces that were thermally isolated from building enclosures.

Our coupling approach, that will be described in the following sections, is closer to the latter approach in the sense that a reduced order indoor air model is obtained from a detailed CFD model. However it could be generally applicable for the evaluation of building control algorithms due to a natural interface to an envelope model. It belongs to neither internal nor external coupling strategies since a CFD software is not directly utilized in a simulation. Compared to the FFD, this approach emphasizes more on computation speed than accuracy in order to enable a weekly/monthly simulation. However it will be shown that the coupled model is also sufficiently accurate based on the comparison to experiments (See Section 4.3). 


\subsection{Development of a ROM-CFD coupled model}

Let's consider the following nonlinear dynamic equations.

$$
\begin{aligned}
\dot{x}_{1}(t) & =f_{1}\left(x_{1}(t), x_{2}(t), u(t)\right) \\
\dot{x}_{2}(t) & =f_{2}\left(x_{1}(t), x_{2}(t), u(t)\right) \\
z(t) & =g\left(x_{1}(t), x_{2}(2), u(t)\right)
\end{aligned}
$$

where $x_{1}(t) \in \mathbb{R}^{n_{1}}, x_{2}(t) \in \mathbb{R}^{n_{2}}, u(t) \in \mathbb{R}^{m}$ and $z(t) \in \mathbb{R}^{p}$ for all $t \in \mathbb{R}^{+}$. We assume each function, $f_{1}$ and $f_{2}$, is continuously differentiable. Define a fixed operating point, $\left(x_{1, o}, x_{2, o}, u_{o}\right)$, and let $\delta x_{i}(t)=x_{i}(t)-x_{i, o}$ and $\delta u(t)=u(t)-u_{o}$. From a nonlinear model, one can generate the following affine model at the operating point $\left(x_{1, o}, x_{2, o}, u_{o}\right)$.

$$
\begin{aligned}
\dot{\delta x_{1}} & \approx f_{1}\left(x_{1, o}, x_{2, o}, u_{o}\right)+\mathbb{A}_{1,1} \delta x_{1}+\mathbb{A}_{1,2} \delta x_{2}+\mathbb{B}_{1} \delta u \\
\delta \dot{x_{2}} & \approx f_{2}\left(x_{1, o}, x_{2, o}, u_{o}\right)+\mathbb{A}_{2,1} \delta x_{1}+\mathbb{A}_{2,2} \delta x_{2}+\mathbb{B}_{2} \delta u \\
\delta z & \approx \mathbb{C}_{1} \delta x_{1}+\mathbb{C}_{2} \delta x_{2}+\mathbb{D} \delta u
\end{aligned}
$$

where $\delta z(t)=z(t)-g\left(x_{1, o}, x_{2, o}, u_{o}\right), \mathbb{A}_{i, j}=f_{i, j}\left(x_{1, o}, x_{2, o}, u_{o}\right), \mathbb{B}_{i}=f_{i, u}\left(x_{1, o}, x_{2, o}, u_{o}\right)$, $\mathbb{C}_{j}=g_{, j}\left(x_{1, o}, x_{2, o}, u_{o}\right), \mathbb{D}=g_{, u}\left(x_{1, o}, x_{2, o}, u_{o}\right)$ and $\left(f_{i, j}, f_{i, u}, g_{, j}, g_{, u}\right)$ are the $J a$ cobian matrices of $\left(f_{i}, f_{i}, g, g\right)$ with respect to $\left(x_{j}, u, x_{j}, u\right)$ evaluated at the operating point, $\left(x_{1, o}, x_{2, o}, u_{o}\right)$. The time dependency of each variable is omitted in the expression.

Equations 1 and 2 represent linearized models for the dynamics of a building envelope and indoor air environment, respectively. Our target is to construct $\left\{f_{i}\left(x_{1, o}, x_{2, o}, u_{o}\right), g\left(x_{1, o}, x_{2, o}, u_{o}\right), \mathbb{A}_{i, j}, \mathbb{B}_{i}, \mathbb{C}_{j}, \mathbb{D}\right\}_{i, j \in 1,2}$ such that Eqn. 1 describes the conduction through walls/ windows, radiative interaction between inside walls, radiative interaction between external walls and sky/ground, convective heat exchange between external walls and outdoor air, while Eqn. 2 describes mass, momentum and energy balances for the indoor air domain. Furthermore, we want the state dimension for the indoor air dynamics, $n_{2}$, as small as possible while preserving input-output responses.

Our strategy for model construction has the following main steps.

1. Defining inputs, outputs (I/O) and coupled variables between CFD and building envelope models

2. Development of a linear time invariant (LTI) state space model for the building envelope

3. Calculation of a nominal boundary condition where the CFD model will be linearized

4. Data generation by perturbing the CFD model at the nominal condition and generation of a LTI reduced-order model (ROM)

5. Aggregation of the two models 
The first step is important and may differ for different systems of interest. The CFD model should respond to heat/latent sources inside its domain and should have wall surface temperatures and supply air conditions as boundary conditions. The envelope model must react to weather conditions and heat flux distributions at the wall surfaces. Therefore, it is natural to define wall temperatures and convective heat fluxes to the walls as coupling variables. An example list of $\mathrm{I} / \mathrm{O}$ is shown in Table 1 in Section 3.2.1.

Zhai, Chen, Haves, and Klems [23] summarized three main difficulties for CFD-BES coupling. The first is thermal response time scale discrepancy where the building structure has a time scale of hours to a day but the zone air has a seconds to minutes time scale. The second difficulty is simulation time discontinuities: the execution time for BES is on the order of a few seconds per zone per year simulation period but CFD may take hours for a several day simulation depending on the resolution of the grid. The last issue is spatial resolution discrepancy: CFD requires dense mesh resolution near the boundary between the air and wall while BES requires only a few nodes per a wall. In order to address the first issue, continuous time dynamic models are preferred over discrete models for the two different physical domains because; 1) they allow use of numerically efficient adaptive time-step ordinary differential equation (ODE) solver algorithms for the stiff problem and 2) it is straightforward to integrate the building envelope and indoor air models. The reasons should become clear following Section 2.5. The second issue is handled through the use of a reduced-order indoor air model. The third issue is addressed by the choice of discretization in the baseline envelope model used to generate a reduced-order model.

\subsection{Development of LTI Building Envelope Model}

This step involves construction of a state space representation of the building structure, i.e. walls and windows, which responds to indoor and outdoor air conditions, based on the defined I/O and coupling variables. The natural way to treat the coupling variables is to define the convective heat fluxes as inputs and the internal wall temperatures as outputs for the envelope model.

Our form is (See Appendix 7.1. for details):

$$
\begin{aligned}
\dot{x_{1}}(t) & =A_{1} x_{1}(t)+F_{1} v_{1}(t)+B_{1} u_{1}(t) \\
y_{1}(t) & =G_{1} x_{1}(t)
\end{aligned}
$$

where the input $v_{1}$ represents the convective heat flux provided by the reduced-order CFD model, and the input $u_{1}$ represents several exogenous terms, such as solar irradiation and outdoor air temperature, followed by predefined input lists. The state $x_{1}$ represents temperatures at wall nodes. The output vector $y_{1}$ contains the inside wall temperatures and provides boundary conditions for the reduced-order CFD model. Thus the map $v_{1}(t) \mapsto y_{1}(t)$ represents Neumann boundary condition (BC) to Dirichlet BC. The following conditions are utilized within our framework. 
- Continuous time domain.

- Individual wall elements can be divided into sub elements to facilitate coupling to the CFD indoor air model. This is a requirement to overcome the spatial resolution discrepancy between CFD and BES. This is also appropriate when surface temperatures along a wall vary significantly, possibly due to solar or other nonuniform inputs.

- Longwave radiative interactions between enclosure surfaces of zone air are treated in the envelope model. Although commercial CFD packages include detailed radiation equation solvers, such as ray tracing [24], radiant exchange is treated in the envelope model to decrease computational demands on the CFD.

In order to focus on the overall coupling procedure, the detailed formulation of the state-space building envelope model is presented in the Appendix 7.1. The main advantage of this formulation is that the system matrices needed for a model aggregation to the reduced-order indoor model can be automatically generated based on building geometry and properties of layers and windows.

\subsection{Development of LTI Reduced-Order indoor CFD model}

The approach to developing a reduced-order indoor CFD model is to construct a linear time-invariant (LTI) model that approximates the input-output behavior observed in computational fluid dynamic (CFD) simulations. Accordingly, a CFD model was developed based on a commercial CFD tool. There are a number of approaches to extract reduced-order LTI models from response data (system identification), depending on the combination of a model structure and an identification algorithm $[25 ; 26]$. For example, one may choose an autoregressive exogenous (ARX) model structure and least-squares training algorithm, or another may select a general state space model structure with a subspace identification method [27], e.g. N4SID. The goal in this study is to find a low order model that is computationally efficient. A constrained model structure in a state space description is used to reduce the number of parameters to be estimated. The structure is presented in Section 7.2.2.

Because of the nonlinearities embedded in the dynamics of zone air associated with mass, momentum and energy balances, an operating point needs to be defined where the linearly approximated model is developed. The following steps are chosen:

1. Define an operating condition for zone air temperature, e.g. thermostat setpoint, and nominal conditions for weather, denoted as $u_{1, o}$, gains and supply air temperatures (or flow rate). Add a zone air energy balance equation to Eqn. 3.

2. From the combined model, which leads to a typical well mixed model, calculate an operating point, denoted as $x_{1, o}$, under the defined condition of step 1. From $x_{1, o}$, calculate a nominal heat removal rate and wall surface temperatures, denoted as $y_{1, o}:=G_{1} x_{1, o}$ (See Eqn. 3). 
3. Set the internal wall surface temperatures from step 2 and nominal supply air conditions, denoted as $u_{2, o}$, as CFD boundary conditions.

4. Run the indoor CFD model until the simulation results converge and define the converged state together with the nominal conditions as $x_{2, o}$. Also define $y_{2, o}$ and $z_{2, o}$ as nominal convective heat fluxes and nominal CFD outputs of interests.

The concept associated with steps 1 to 3 is similar to a peak load calculation in a BES tool and we use a typical meteorological year (TMY) data. Note that the calculated point $\left(x_{1, o}, x_{2, o}, u_{1, o}, u_{2, o}\right)$ may not to be an equilibrium point. In order the point to be an equilibrium point, it would be necessary to iterate between the building envelope and CFD models to determine converged wall surface temperatures and heat fluxes. However, it would be difficult to implement and would require significant computation. Instead, $\left(x_{1, o}, x_{2, o}, u_{1, o}, u_{2, o}\right)$ can be thought as an estimation of an equilibrium point. Because it is not an equilibrium point, the additional the affine terms in Eqn. 1 and 2 need to be determined.

At the converged CFD state and the nominal values, each input is adjusted with a certain perturbation level in the CFD model and the corresponding output responses are recorded. After applying a parameter estimation method, one can come up with the following form.

$$
\begin{aligned}
\delta \dot{x}_{2}(t) & =A_{2} \delta x_{2}(t)+F_{2} \delta v_{2}(t)+B_{2} \delta u_{2}(t) \\
\delta y_{2}(t) & =G_{2} \delta x_{2}(t)+H_{2} \delta v_{2}(t) \\
\delta z_{2}(t) & =C_{2} \delta x_{2}(t)
\end{aligned}
$$

and

$$
\begin{array}{ll}
\delta v_{2}(t)=v_{2}(t)-v_{2, o}, & \delta u_{2}(t)=u_{2}(t)-u_{2, o} \\
\delta y_{2}(t)=y_{2}(t)-y_{2, o}, & \delta z_{2}(t)=z_{2}(t)-z_{2, o}
\end{array}
$$

where the input $v_{2}$ represents the internal wall temperature provided by the envelope model (i.e. $v_{2}=y_{1}$ in Eqn. 3), and the input $u_{2}$ represents several exogenous terms, such as internal convective heat gain and latent gain. The output $y_{2}$ contains the heat fluxes from zone air to walls (i.e. $y_{2}=v_{1}$ in Eqn. 3), to complete the coupling (thus the map, $v_{2} \mapsto y_{2}$, indicates Dirichlet to Neumann BC). The exogenous output, $z_{2}$, contains additional quantities of interest such as local zone air conditions (e.g. temperature and humidity ratio) and return air conditions (temperature, flow rate and humidity ratio). The $v_{2, o}, u_{2, o}, y_{2, o}, z_{2, o}$ indicates the wall boundary conditions, the supply air conditions, the heat fluxes and the exogenous outputs all evaluated at the nominal condition described above.

\subsection{Model Aggregation and Further Model Order Reduction}

To complete the construction of coefficients in Eqn. 1, 2 and 3, the coupling variables, $\left(y_{1}, y_{2}\right)$, need to be eliminated. This aggregation process is important 


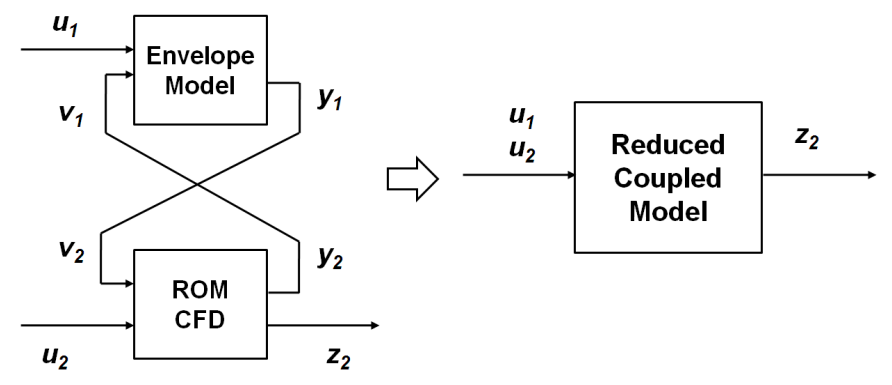

Figure 1: Model Aggregation

when a detailed analysis, e.g. investigation of eigenvalues of the integrated system or propagation of input uncertainty as discussed in Section 4, is required or the coupled model is used for a state observer, a disturbance estimator or a model-based controller. However this process may be omitted when it is used only as a simulation platform in the analysis and design of a controller. The integration procedure is graphically shown in Fig. 1 and a composition formula for the interconnection of the two systems is shown below.

Applying $\delta y_{2}=y_{2}-y_{2, o}, \delta u_{2}=u_{2}-u_{2, o}$ and $\delta v_{2}=v_{2}-v_{2, o}$ in Eqn. 4 results in

$$
\begin{aligned}
& \dot{\delta x_{2}}=A_{2} \delta x_{2}+F_{2}\left(v_{2}-v_{2, o}\right)+B_{2}\left(u_{2}-u_{2, o}\right) \\
& y_{2}=G_{2} \delta x_{2}+H_{2}\left(v_{2}-v_{2, o}\right)+y_{2, o} \\
& z_{2}=C_{2} x_{2}+z_{2, o} .
\end{aligned}
$$

Since $v_{1}=y_{2}, v_{2}=y_{1}$ and $y_{2}=\left(G_{2} \delta x_{2}+H_{2} G_{1} x_{1}-H_{2} v_{2, o}+y_{2, o}\right), y_{1}=G_{1} x_{1}$,

$$
\begin{aligned}
& \dot{x}_{1}=A_{1} x_{1}+F_{1}\left(G_{2} \delta x_{2}+H_{2} G_{1} x_{1}-H_{2} v_{2, o}+y_{2, o}\right)+B_{1} u_{1} \\
& \dot{\delta} x_{2}=A_{2} \delta x_{2}+F_{2} G_{1} x_{1}+B_{2} u_{2}-B_{2} u_{2, o}-F_{2} v_{2, o} \\
& z_{2}=C_{2} x_{2}+z_{2, o} .
\end{aligned}
$$

Rearranging the equations results in

$$
\begin{aligned}
{\left[\begin{array}{c}
\dot{x}_{1} \\
\delta \dot{x}_{2}
\end{array}\right] } & =\left[\begin{array}{cc}
A_{1}+F_{1} H_{2} G_{1} & F_{1} G_{2} \\
F_{2} G_{1} & A_{2}
\end{array}\right]\left[\begin{array}{c}
x_{1} \\
\delta x_{2}
\end{array}\right]+\left[\begin{array}{cc}
B_{1} & 0 \\
0 & B_{2}
\end{array}\right]\left[\begin{array}{l}
u_{1} \\
u_{2}
\end{array}\right]+\left[\begin{array}{c}
-F_{1} H_{2} v_{2, o}+F_{1} y_{2, o} \\
-B_{2} u_{2, o}-F_{2} v_{2, o}
\end{array}\right] \\
z_{2} & =\left[\begin{array}{ll}
0 & C_{2}
\end{array}\right]\left[\begin{array}{c}
x_{1} \\
\delta x_{2}
\end{array}\right]+z_{2, o} .
\end{aligned}
$$

Due to the affine terms, a composition formula is slightly different than the star-product formula [28; 29], but the derivation procedure is the same. 


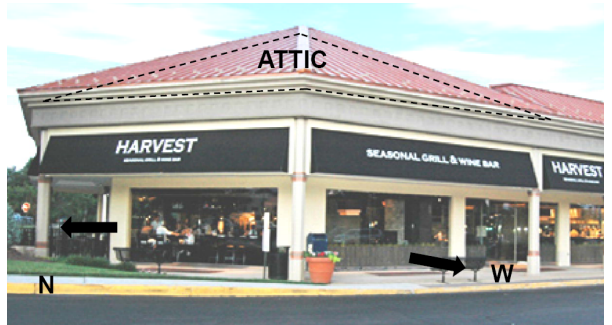

(a) External View

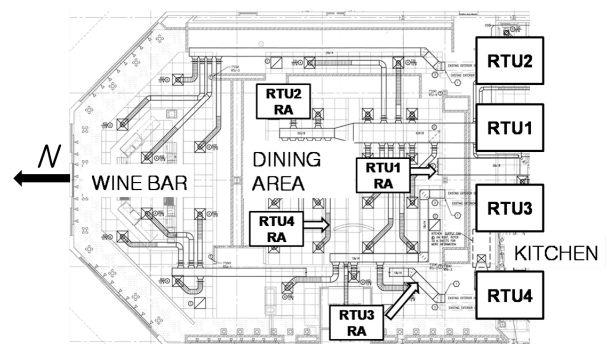

(b) Inner View and Locations of return air vent (RA)

Figure 2: Descriptions of Case Study Building

Eqn. 6 corresponds to the set of coefficients $\left\{\mathbb{A}_{i, j}, \mathbb{B}_{i}, \mathbb{C}_{j}, \mathbb{D}, f_{i}\left(x_{1, o}, x_{2, o}, u_{o}\right)\right.$, $\left.g\left(x_{1, o}, x_{2, o}, u_{o}\right)\right\}_{i, j \in 1,2}$, thus the target is achieved.

After constructing the aggregated model, further reduction of model order is possible. The balanced-truncation method [30] may be applied to eliminate subspaces of a state space that are both hard to observe and hard to reach to get further reduction of the aggregated state dimension. For simulation purposes, adaptive time step ODE solver algorithms, such as Dormand-Prince RK4-5 pair on Matlab's ode45, can be used.

\section{CASE STUDY: DEVELOPEMENT OF A REDUCED-ORDER CFD COUPLED MODEL FOR A SMALL COMMERCIAL BUILD- INGS}

\subsection{Case Study Building Description}

A restaurant in suburban Philadelphia (see Fig. 2a) is a demonstration site for the DOE supported Consortium for Building Energy Innovation (CBEI). Some important points are:

- Building interior dimensions: $23.34 \mathrm{~m}$ (70 ft) width, $19.82 \mathrm{~m}(65 \mathrm{ft})$ depth and $3.35 \mathrm{~m}$ (11 ft) height.

- Four roof top units (RTUs) serve the dining and wine bar area in Fig. 2b.

- North and West surfaces are windows while the East surface is a wall. Kitchen area is located at the South but is outside of our computation domain.

\subsection{Developement of a Reduced-Order CFD Coupled Model}

\subsubsection{Definition of $I / O$ and coupling variables}

Input/output lists for this case study building are shown in Table. 1. The variables in the 2 nd and 3 rd rows belong to the ROM-CFD and envelope models 
Table 1: Definition of Inputs/Outputs and Coupled Variables for Construction of Reduced-Order CFD Coupled Model $(T, \phi$ and $\dot{m}$ represent temperatures, humidity ratio and mass flow rates respectively.)

\begin{tabular}{|l|l|l|l|l|}
\hline & Exogenous Inputs & Coupling Inputs & Exogenous Outputs & Coupling Outputs \\
\hline $\begin{array}{l}\text { ROM- } \\
\text { Model }\end{array}$ & $\begin{array}{l}\text { Internal gain (con- } \\
\text { vective part, latent) }\end{array}$ & $\begin{array}{l}\text { Internal temp. of } \\
\text { each surface }\end{array}$ & $\begin{array}{l}\text { Local and volume } \\
\text { averaged zone air } \\
\text { condition }(\mathrm{T}, \phi) \\
\text { Return air condi- } \\
\text { tion }(T, \phi, \dot{m})\end{array}$ & $\begin{array}{l}\text { Convective heat } \\
\text { flux on each surface }\end{array}$ \\
\hline $\begin{array}{l}\text { Envelope } \\
\text { Model }\end{array}$ & $\begin{array}{l}\text { Internal gain (ra- } \\
\text { diative part) } \\
\text { Temp. outdoor air } \\
\text { Temp. ground/sky } \\
\text { Solar radiation on } \\
\text { surfaces }\end{array}$ & $\begin{array}{l}\text { Convective heat } \\
\text { flux on each surface }\end{array}$ & & $\begin{array}{l}\text { Internal temp. of } \\
\text { each surface }\end{array}$ \\
\hline
\end{tabular}

respectively. Both models share convective heat fluxes and internal wall building surface temperatures as coupling variables. In this study, each unique wall and window orientation was assumed to be at a uniform, area-averaged temperature at any given time. The return air temperatures are included in the output list of the CFD model and are compared with measured values.

\subsubsection{Development of LTI Building Envelope Model}

Based on building geometry information and wall/window properties, a LTI envelope model for the site was developed. A method described in the Appendix was utilized to construct the LTI model. Two to four nodes per material layer were assigned along a wall thickness. Kim and Braun ([31; 32]) provide validation of the approach through comparisons with existing BES tools (Modelica Building's Library and TRNSYS) for several buildings.

\subsubsection{Development of LTI Reduced-Order Indoor CFD Model}

A commercial CFD code was used to generate data recording the relevant responses (wall fluxes, etc) to step-like inputs (wall temperatures, etc.). The data generation and LTI model construction are included in the Appendix. We refer readers to [33] for comparisons of results of the LTI reduced-order indoor air model to the full order CFD model and detailed parameter settings, e.g. full descriptions of inputs and outputs of the CFD model and the perturbation level for each input. 


\section{CASE STUDY: ANALYSIS, VALIDATION AND APPLICATION OF THE COUPLED MODELING APPROACHES}

This section considers the case study restaurant and presents comparisons of model predictions with measurements and demonstration of the integrated tool for evaluating the impact of thermostat location on comfort and energy usage. The section begins with an analysis of the model structure to highlight the different time scales associated with the walls and indoor air. In addition, the computational requirements of the model are evaluated in order to demonstrate the usefulness of the model in evaluating performance over long periods of time (e.g., months). The experimental validation includes an analysis of the uncertainty in the output predictions due to input uncertainties associated with the case study.

\subsection{Model analysis}

After construction of the two models, they are aggregated based on Eqn. 6 in Section 2.5. The structure of the $\mathbb{A}$-matrix is shown in Fig. 4.1 in which the first block, size of $35 \times 35$, and the last block, size of $22 \times 22$, correspond to the states of the envelope and the reduced-CFD respectively. Because no model order reduction method was applied on the envelope side, the state dimension for the envelope model is higher than the reduced-order air model. Further model order reduction either on the envelope model or combined model is possible, via e.g. balanced truncation method, but was not employed in our case study, because it was fast enough (See Section 4.2). The tri-diagonal structure is due to finite element descriptions as shown in Eqn. 19 for the first block. The full square matrix corresponding to $19-26^{\text {th }}$ states appears due to the long wave interaction between internal walls (See $\tilde{H}_{r a d, i n}$ in Eqn. 17.). A diagonal matrix for the second block is shown due to Eqn. 24. The spectrum for the $\mathbb{A}$-matrix ranges from $-1.76 \times 10^{-5}(1 / \mathrm{sec})$ to near zero. This indicates that the coupled model describes distinct time scale dynamics. Based on the eigenvector analysis, the slowest dynamics corresponds to a wall temperature node of a concrete floor. Furthermore the system model is exponentially stable due to real values of all poles that are located in left half plain.

\subsection{Numerical Performance}

Table 2: Computation time for a reduced-order coupled CFD model under a feedback control [sec]

\begin{tabular}{|c||c|}
\hline Simulation duration & Computation Time \\
\hline 1 day & 0.572 \\
\hline 10 days & 9.026 \\
\hline 30 days & 30.345 \\
\hline
\end{tabular}




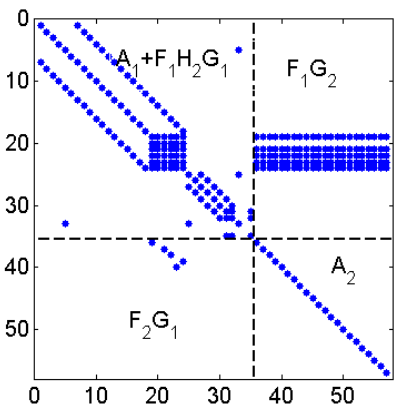

(a) Structure of $\mathbb{A}$-matrix

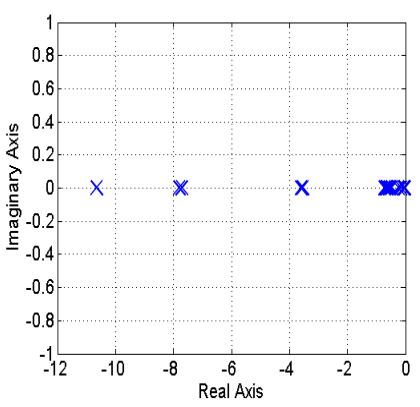

(b) $\mathbb{A}$-matrix spectrum $(1 / \mathrm{sec})$

Figure 3: Analysis of a coupled model

In order to evaluate numerical performance of the reduced-order coupled CFD model, simulation time was determined for the model implemented within the Simulink environment and running on a $3.1 \mathrm{GHz}$ desktop computer. Table 2 shows computation time of the closed-loop system consisting of the model and conventional thermostats. The control logic is such that if a thermostat temperature is higher than its set-point, then the RTU turns on until the temperature falls below the set-point minus a deadband. The computational times are based on ode15s, which is a stiff ODE solver of MATLAB Version 7.10 (R2010a). The results demonstrate that the coupling approach makes it feasible to use a reduced-order coupled CFD model for control analysis purposes.

\subsection{Comparisons with Experimental Results}

\subsubsection{Experimental set-up}

For the validation, measured input data was selected and provided to the model. Corresponding model outputs were recorded to compare to measured outputs. As inputs, the supply air temperature for each RTU and the outdoor air temperature were selected. Due to technical issues (e.g. low sampling rate, resolution and dynamics of thermostat sensors), the return air temperatures to the RTUs were selected as outputs instead of thermostat temperatures. The locations of return air vents for the RTUs are shown Fig. 2b and corresponding return air temperature sensors are located at the upstream inlet of the RTUs (dashed circles). Supply air temperature sensors are located downstream of the units and are shown with filled circles in the same figure (The outdoor air sensor is not shown). All temperature sensors are T-Type thermocouples with less than $0.1{ }^{\circ} \mathrm{C}$ expanded uncertainty at ambient temperatures [34, p. 14].The sampling period of the sensors is one minute.

\subsubsection{Sources of uncertainties}

The experiments were set up on an existing building and it is important to discuss sources of uncertainties in the measurement data before discussion of 
model validation. Many of the disturbances, such as infiltration or exfiltration and internal gains due to occupant and cooking loads are unmeasurable. In addition, the kitchen, as shown in Fig. $2 \mathrm{~b}$ and 16, that is outside of our computation domain, could act as unknown heat source or sink. The restaurant is open between 11:00 AM and midnight and during this time the occupancy and internal gains are highly variable and uncertain, especially around lunch and dinner. An uncertainty analysis with respect to the unmeasured disturbances during this time period is very difficult and may not reliable because of lack of information. In order to minimize the effects of unknown occupancy and allow fairer comparisons, validation was mostly carried out during unoccupied periods.

The coupled model requires air flow rate as an input for each supply diffuser, which significantly affects the model behavior. Continuous measurements of air flow were not available due to high sensor costs and a large number of diffusers. However, the system uses constant speed fans and the air flow is constant whenever the fans operate. Therefore, one time measurements of air flows for the diffusers were determined using a hot-wire anemometer. The anemometer was moved from one diffuser to another in order to determine the air flows during fan operation. Three of the RTUs have continuous air flow throughout the day. However, the largest RTU cycles the fan on and off with the compressor cycling. The uncertainties in the model predictions are largely driven by large uncertainties in the air flow.

Therefore, we only perform an uncertainty quantification with respect to the air flow rates. In this case, we quantify how the uncertain flow rate inputs propagate to the outputs. In other words, we assigned uncertain elements, denoted as $\delta m(t) \in \mathbb{R}^{4}$, on the air flow inputs and compute confidence intervals of the model outputs. Since the coupled model is linear time invariant, this can be done analytically.

Consider a discrete LTI system ${ }^{1}$, denoted as $G(z)$ for its transfer function. Given an input sequence denoted as $u(n) \in \mathbb{R}^{m}$, denote the corresponding output as $y(n) \in \mathbb{R}^{p}$. When a sequence, $\delta u(n)$, is added to the inputs, $u(n)$, the response will also deviate from the $y(n)$. Let $\delta y(n)$ be the deviated response from the $y(n)$ due to the additional input sequence $\delta u(n)$. Clearly $\delta Y(z)=G(z) \delta U(z)$ due to the linearity of $G$, where $\delta U(z)$ and $\delta Y(z)$ are z-Transforms of $\delta u(n)$ and $\delta y(n)$, respectively.

Suppose the $\delta u(n)$ is a white Gaussian noise process with the covariance matrix of $E\left[\delta u(n) \delta u(n)^{T}\right]=Q$. We are interested in the covariance matrix of

\footnotetext{
${ }^{1}$ The same analysis for a continuous time system is possible. However it is more reasonable to assume the uncertain processes, $\delta u(n)$, of the mass flow rates is piece-wise constant for a period of time. Note the assumption of $\delta u(n)$ as a continuous Gaussian random process will lead to high frequency oscillation of the flow rate even within several seconds.
} 
$\delta y$ due to the $\delta u$. Then the following relationship holds (See [35][pp.44-45] for details.)

$$
\gamma_{\delta y}(0)=\frac{1}{2 \pi} \int_{0}^{2 \pi} G\left(e^{j w}\right) Q G^{\dagger}\left(e^{j w}\right) d w
$$

where $\gamma_{\delta y}(\cdot)$ is the auto correlation function of $\delta y$, i.e. $\gamma_{\delta y}(k)=E[\delta y(n) \delta y(n+$ $\left.k)^{T}\right]$ and $\dagger$ denotes conjugate transpose. If the system $G$ is exponentially stable, then the integration term can be obtained analytically by solving a Lyapunov equation as follows; Let (A,B,C,D) be a realization $G$. Then

$$
\begin{aligned}
\frac{1}{2 \pi} \int_{0}^{2 \pi} G\left(e^{j w}\right) Q G^{\dagger}\left(e^{j w}\right) d w & =C P C^{T}+D Q D^{T} \\
A P A^{T}-P & =-B Q B^{T} .
\end{aligned}
$$

The formula can be easily derived based on Parseval's theorem and is omitted (See $[29 ; 36]$ for detail derivation). The integration is the covariance matrix of the output because $E\left[\delta y(n) \delta y(n)^{T}\right]=\gamma_{\delta y}(0)$.

In our case, the outputs, $y$, are the return air temperatures and the inputs, $u$, are the supply air mass flow rates. A discrete time LTI system, $G$, in Eqn. 7 can be readily obtained from Eqn. 6 and exponential stability can be verified ${ }^{2}$. We assigned $10 \%$ of each nominal flow rate as the uncertainties. The diagonal elements of the calculated covariance matrix of the return air temperatures, i.e. $\left(\gamma_{\delta y}(0)\right)_{i, i}, i \in\{1,2,3,4\}$ in Eqn. 7 , are $(0.03,0.10,0.06,0.05)\left({ }^{\circ} C^{2}\right)$, which correspond to the standard deviation (s.t.d.) of $(0.18,0.31,0.25,0.23)\left({ }^{\circ} C\right)$. For the following results, $99.6 \%$ confidence intervals (C.I.), which corresponds to three times of the s.t.d., will also be shown in the coupled model validation. It is important to remember that the confidence intervals account for uncertain flow rates not for the unmeasured disturbances.

\subsubsection{Validation of the coupled modeling approach}

We performed comparisons between CFD outputs and measurements for several data sets and some of the resulting comparisons are presented. All the comparisons have been done only for the unoccupied period to eliminate uncertain disturbances due to occupants, cooking and solar loads as much as possible. For CASE 1, a step like test was performed during the unoccupied period: each RTU compressor was set to ON or OFF for different time intervals. The data set for model validation is shown in Fig. 4. The points of sudden supply air temperature drops, e.g. 02:00 AM, indicate the time when RTU compressors

\footnotetext{
${ }^{2}$ The zero-order holder equivalent discrete system with one-hour time step was used for the discretization, assuming that the uncertain process of the mass flow rates is constant for the period of time. Based on a parametric study, the calculated covariance matrices were not sensitive to the choice of a time step.
} 
start to work. We divided the data into two parts: before and after 6:00 AM. For part 1, before 6:00 AM, the outdoor air temperature was almost constant at around $18^{\circ} \mathrm{C}$ and the corresponding responses to RTU operation were dominated by the dynamics of the indoor air. This data was considered suitable for checking the response of coupled model air dynamics. Meanwhile, after 6:00 AM to noon, outdoor air temperature increased rapidly with a rate of about $3^{\circ} \mathrm{C} / \mathrm{hr}$. Thus, the part 2 was considered useful for checking the responses of the envelope portion in the coupled model.

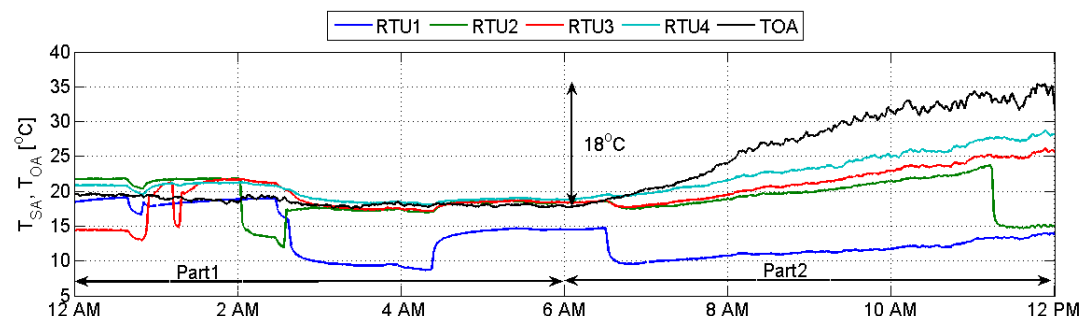

Figure 4: Case 1: Input data for a reduced-order coupled CFD/envelope model

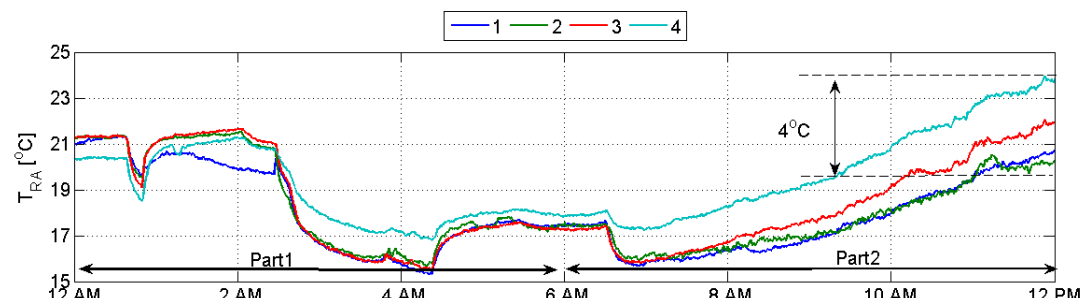

Figure 5: Case 1: Output measurement data for validation of a reduced-order coupled CFD/envelope model

The measurement outputs corresponding to the input data are shown in Fig. 5. Notice that there is significant temperature spatial variations between the zones. It is around $2^{\circ} \mathrm{C}$ on average and reaches up to $4^{\circ} \mathrm{C}$ at $12: 00 \mathrm{PM}$. The output comparisons for the part 1 and 2 are shown in Fig. 6. The blue line represents the output response of the coupled model, whereas the black line is the measured output. The measurements are within the confidence interval (C.I.) for most of the time. In addition, the model properly captures the dynamics due to RTU cycling. However, there is a bias in the predictions especially for RTU3. Some possible explanations include: 1) The CFD model was linearzied at a typical daytime operating condition but the experiment was performed during the night time; 2) The kitchen load discussed in Section 4.3 .2 could exist 

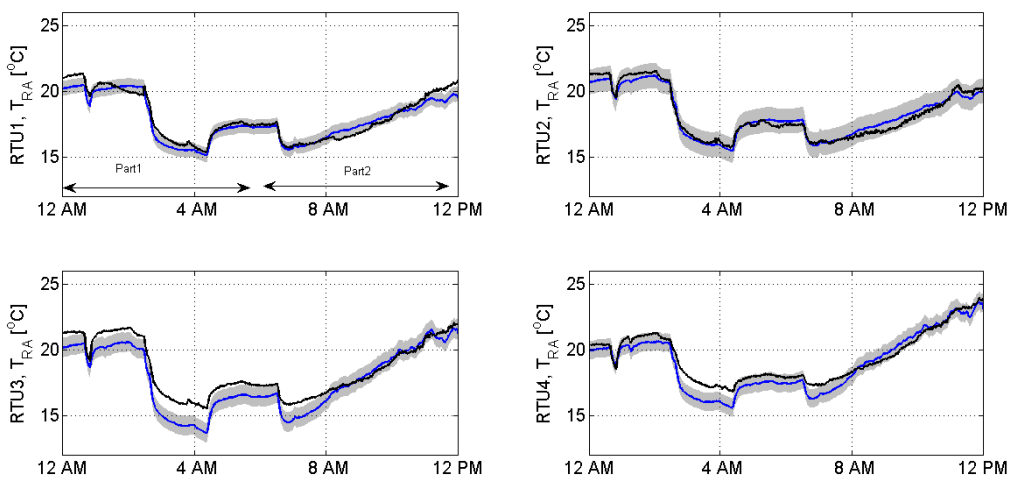

Figure 6: Case 1: Output comparisons for a reduced-order coupled CFD/envelope model. Black: measured, Blue: CFD estimted with nominal flow rates, Grey: 99.6\% confidence interval w.r.t. flow rate uncertainties

even for the night time. 3) The initial conditions may not be well matched. Although the fast air dynamics attenuate the effect of the mismatched condition for the air states within 10-30 min as shown in Fig. 6, the slow dynamics of the building structure could act as pseudo heat sources/sinks for the coupled model if the initial conditions differ significantly. We estimated the initial condition of the coupled model optimally in a least squares (LS) sense but the LS estimator could lead to biased estimation if there is non-zero mean unmeasured disturbances in the data.

Nevertheless, measurements are within the C.I. for most of time. The responses of the model also match well with the measured outputs for the part 2 data, where the main driving force is outdoor air temperature. Therefore it can be concluded the CFD model is reliable and captures the important dynamics of the indoor air.

CASE 2 data provides a useful source to compare the frequency responses for a specific frequency between the true building and the coupled model ${ }^{3}$. One of RTU cycles periodically (about a 30 min period) during the unoccupied period (See Fig. 7). From 12:00 AM to 10:00 AM, the predicted magnitude and phase response reasonably match the measured response shown in Fig 8.

CASE 3 data shown in Fig 9 may be useful to compare dynamics under

\footnotetext{
${ }^{3}$ The missing outdoor air temperature data in Fig. 7 is interpolated for the inputs of the model.
} 


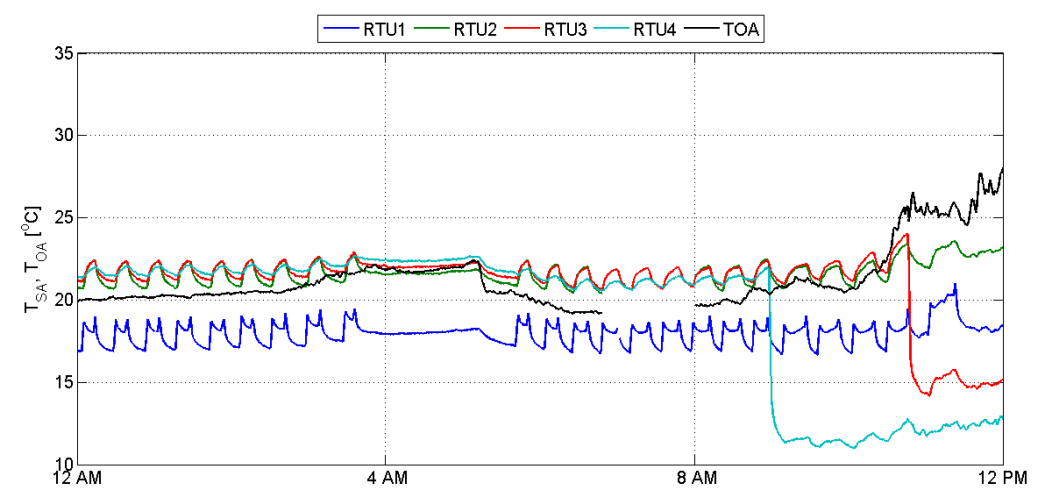

Figure 7: Case 2: Input data for a reduced-order coupled CFD/envelope model

random-like inputs. Note that there is neither a clear pattern of each input, even for outdoor air temperature, nor a clear correlation between inputs. This means inputs are informative or persistently exciting with at least several orders to validate the model for wide frequency range. Measurements are within the confidence interval for most of the unoccupied time. The model captures an abrupt temperature change at around 7:00 AM due to the activation of all RTUs (See Fig. 9).

Based on the comparisons of the step response (CASE 1), the frequency response at a certain frequency (CASE 2) and the random-like input response (CASE 3), it can be concluded the coupled model approximates the dynamics of the true indoor and envelope building system very well.

\subsubsection{Further Discussions}

The coupled model can capture spatial variations of thermal conditions, such as those shown for the return temperatures of RTU4 and RTU1 in Fig. 6. Although their trends are similar, there is about a $4^{\circ} \mathrm{C}$ temperature difference. These differences lead to significant comfort variations that are not predicted with a fully-mixed model. The coupled model was developed to test RTU controllers operating within an open space and is based on linearization at a single operating point. Therefore the model could have unreasonable behavior if the system operates far away from the linearized conditions. Our approach to diminish the concern is to chose the perturbation levels, discussed in Section 7.2.2, to approximate dynamics over a wide range. Although more work is needed to perform a detailed evaluation of model accuracy, the coupled model considered here captures the essential behavior of the air dynamics and spatial variations in a computational efficient manner that enables utilization for long-term sim- 

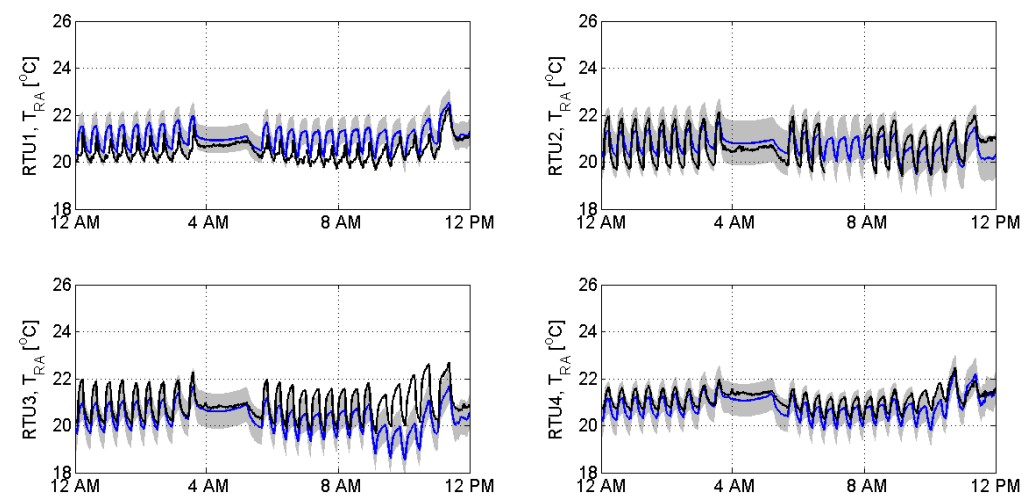

Figure 8: Case 2: Output comparisons for a reduced-order coupled CFD/envelope model. Black: measured, Blue: CFD estimted with nominal flow rates, Grey: 99.6\% confidence interval w.r.t. flow rate uncertainties

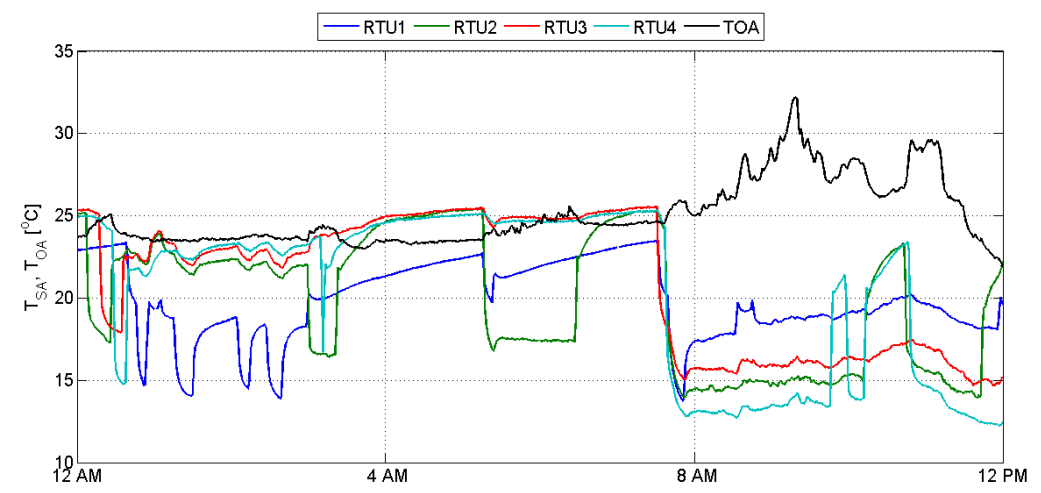

Figure 9: Case 3: Input data for a reduced-order coupled CFD/envelope model 

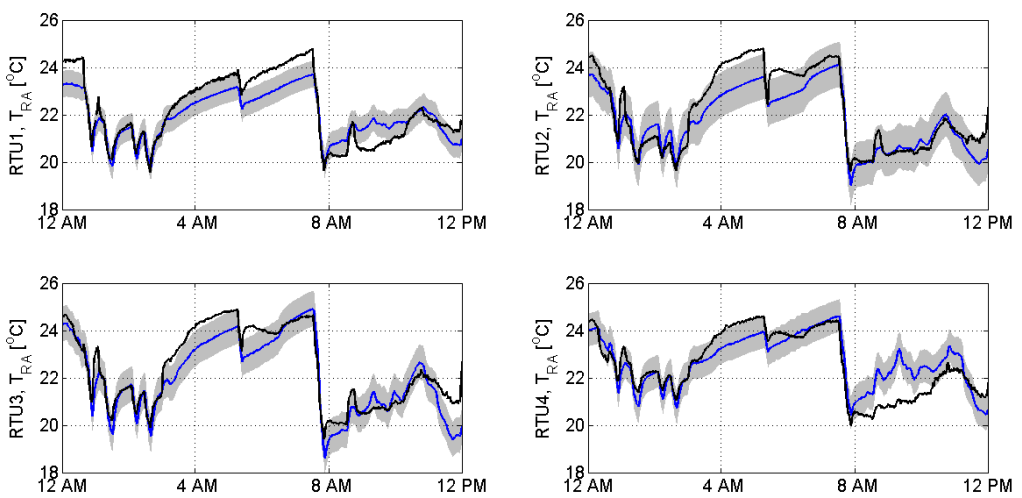

Figure 10: Case 3: Output comparisons for a reduced-order coupled CFD/envelope model. Black: measured, Blue: CFD estimted with nominal flow rates, Grey: $99.6 \%$ confidence interval w.r.t. flow rate uncertainties

ulation.

\subsection{An Application of the Coupled Modeling Approach}

As introduced, the reduced-order CFD coupled modelling approach is targeted to provide a control evaluation test bed for open spaced buildings served by multiple RTUs. Indeed, the coupled model was applied to evaluate several different RTU coordination algorithms $[33 ; 37 ; 38]$. In this section, another application, thermostat location retrofit, is shown and discussed.

\subsubsection{Sensor retrofit analysis}

The problem was to identify causes of poor comfort at the wine-bar area and to suggest a remedy. For practical reasons, installation of several physical sensors at the restaurant where customers are served were limited. After analyzing the restaurant from available data, some important characteristics are found and summarized as follows.

- Four RTUs serve two zones depicted in Fig. 11b, dining (zone 1), wine bar (zone 2). RTU1 and RTU3 serve zone 1 and two RTUs (RTU2 and RTU4) serve zone 2. The supply air diffusers and thermostat locations for these RTUs and zones are shown in Fig. 11a. Note that thermostat locations differ from the return air sensor locations discussed in Section 4.3.

- RTU1 is a larger two-stage unit while the other units are identical and single-stage. The nominal capacities are about 50 and $15 \mathrm{~kW}$ for the former and the latter, respectively. Total COP (coefficient of performance) 


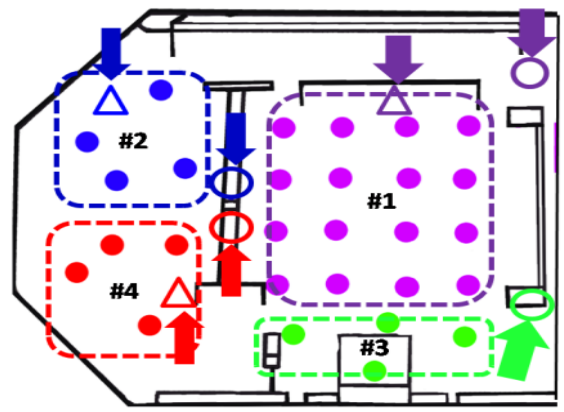

(a) Location of thermostats and supply air diffusers. The filled circles, empty circles and triangles represent the locations of supply air diffusers, current thermostats and proposed thermostats, respectively. The different colors correspond to the different numbered RTUs (\#).

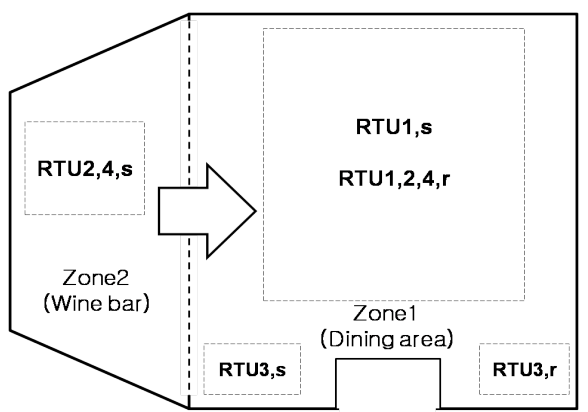

(b) Zone numbering and inter-zonal coupling due to air duct system, where subscripts, $r$ and $s$, represent return air vents and supply air diffusers.

Figure 11: Characteristics of the case study building

of the two-stage RTU (RTU1) is around 35\% higher than the others and its sensible COP is around $50 \%$ higher.

- As shown in Fig. $2 \mathrm{~b}$ and $11 \mathrm{~b}$, there is no return air vent in zone 2. Three of the return vents are located at zone 1. Due to the air duct system, there is significant net flow from zone 2 to zone 1 as depicted in Fig. 11b.

Notice that the thermostats for RTU2 and RTU4, blue and red arrowed circles in Fig. 11a, are close to supply air vents for RTU1 (purple circle) and are likely affected by RTU1 ${ }^{4}$. The thermostat locations seems to be problematic, thereby the effects of change in the sensor locations on control performances were investigated. It is important to emphasize that the thermostat temperatures may not be representative of zone air temperatures due to the non-uniform zone air temperature distributions and poor thermostat locations. The reduced-order CFD coupled model allows investigation of spatial temperature distributions, thereby it is suitable for the study of sensor location retrofit.

A complete simulation requires models for the multiple RTUs and their controllers in addition to the indoor air coupled model. However, the connection between RTU models and the coupled model can be easily achieved, since the supply and return air conditions for the RTUs are defined as inputs/outputs of the coupled model (See Table 1). There is also no difficulty to combine controllers with the building and HVAC models since the sensor positions were

\footnotetext{
${ }^{4}$ Although the thermostats are very close to each other, they are separated by a column (see Fig. 16). Therefore the measured temperature could differ.
} 


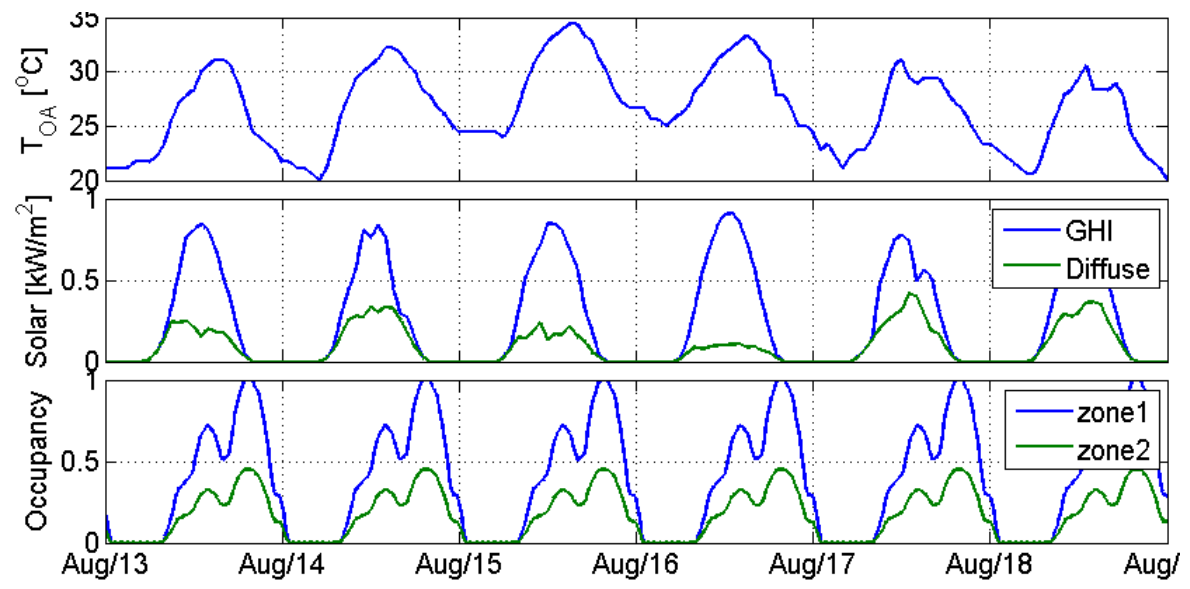

Figure 12: Gain information for a sample period (GHI: horizontal total, Diffues: horizontal diffuse solar radiation)

included as Exogenous Outputs as listed in the Table 1.

Overall simulation details for the complete building model are;

- RTU models were developed based on manufacturer data. The RTU models have $2^{\text {nd }}$ order polynomial forms for total cooling capacity and energy input ratio (EIR). The calculation of sensible and latent capacities are based on the rated sensible heat ratio (SHR), the apparatus dew point and bypass factor approach. Performance degradation due to on/off cycling of the RTUs was considered by including a part load factor. See [39][pp. 565-570] for details..

- Thermostat control law is; if the thermostat temperature is $0.5^{\circ} \mathrm{C}$ higher than its set-point, then it turns on the corresponding RTU. The second compressor of RTU1 is activated when the temperature is $1^{\circ} \mathrm{C}$ above its set-point.

- TMY3 data is used for outdoor air temperature and solar radiation which are inputs for the reduced-order CFD coupled model as shown in Table 1. Fig. 12 shows the weather information and an occupancy schedule for a sample period. Since the wine bar has less seats, around half of the internal gain for the dining zone is assumed.

- All models were integrated in Matlab simulink platform and a stiff ODE solver, ode15s, is used.

One month simulation with the TMY 3 August data was performed. The computation time for the complete building model is about $23.24 \mathrm{sec} /$ day. The slower computation time relative to the results of Table 2 are attributed to the 


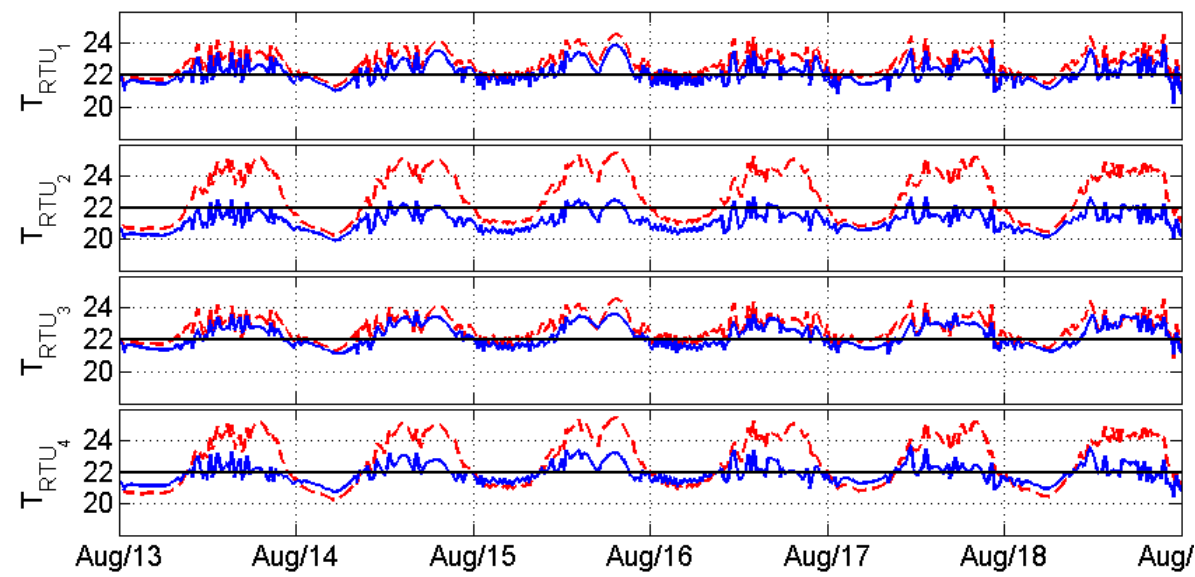

(a) Thermostat (blue solid) and zonal temperature (red dashed) profiles

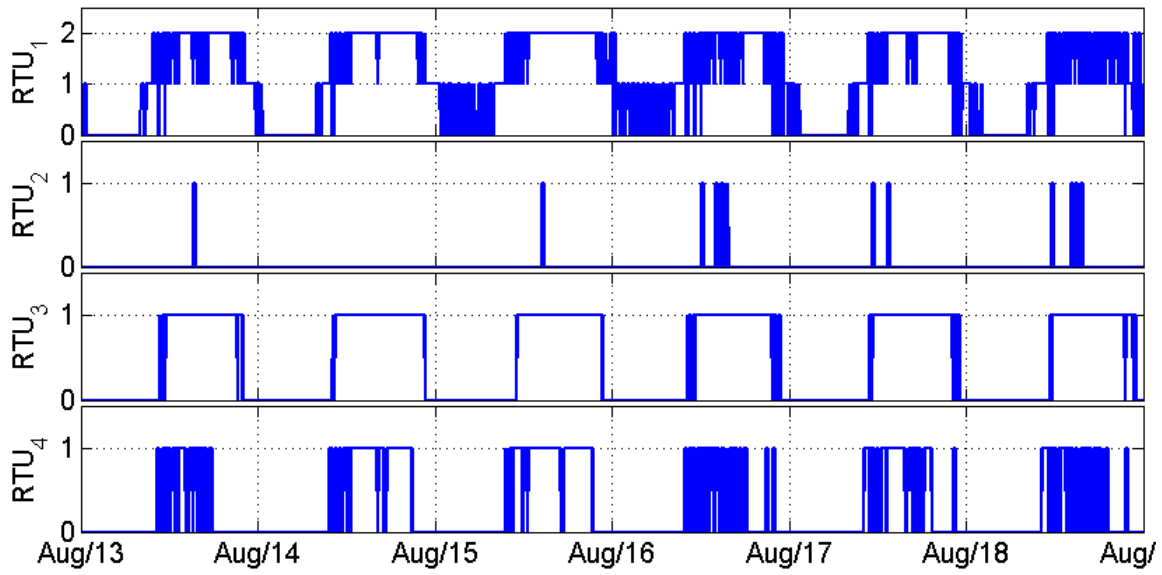

(b) RTU ON/OFF profiles

Figure 13: Control performances before retrofit of stat locations 


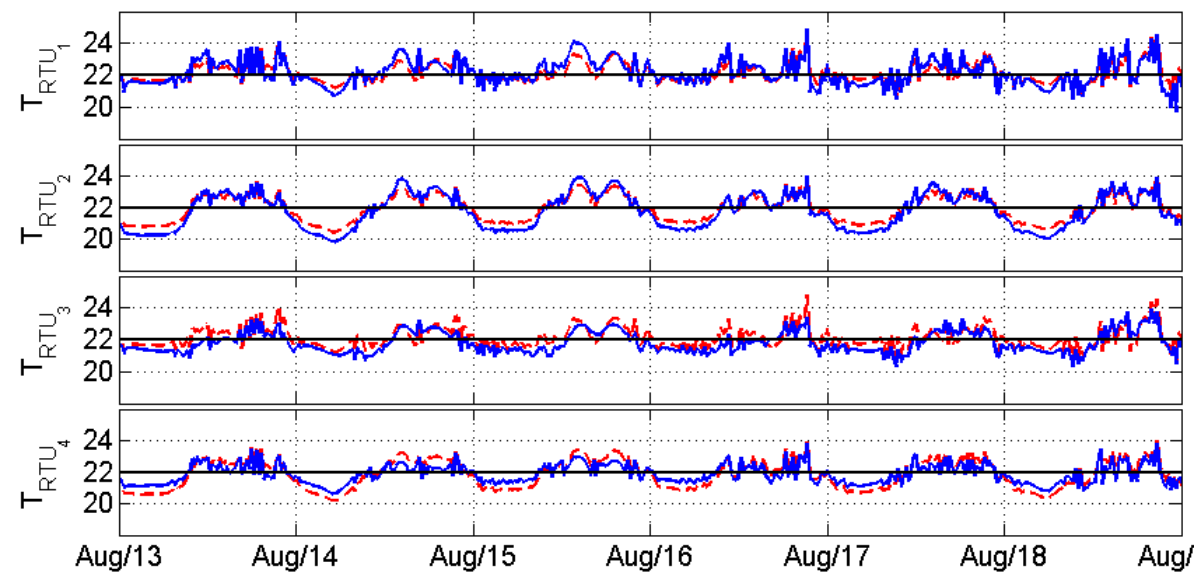

(a) Thermostat (blue solid) and zonal temperature (red dashed) profiles

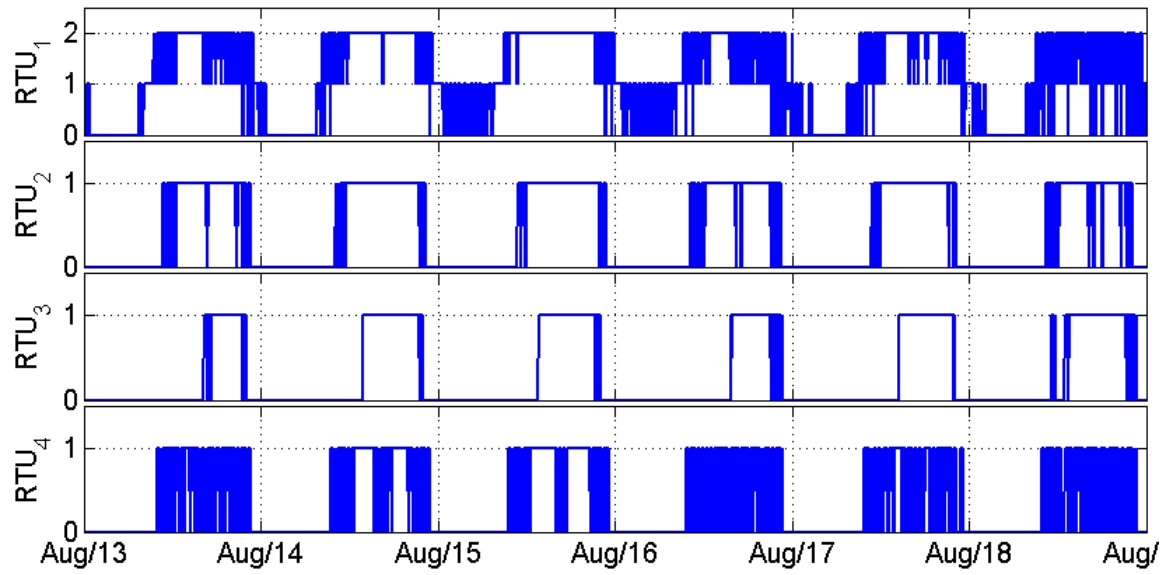

(b) RTU ON/OFF profiles

Figure 14: Control performances after retrofit of stat locations 
inclusion of the RTU models which call several, possibly iterative, psychometric functions and the inclusion of the $\mathrm{ON} / \mathrm{OFF}$ controllers which prevent a longer simulation time step of the adaptive time step solver. Nonetheless the computation time is fast enough for the analysis.

Fig. 13a and 13b show the thermostat temperature profiles and ON/OFF compressor stages under the conventional feedback control with the original thermostat positions for a sample period. Because RTU1 has two separate stages of cooling, it has 3 modes $(0,1$, and 2$)$. In this simulation, no setpoint reset strategy was applied and the set-points for the controls are $22^{\circ} \mathrm{C}$ for all zones. Each thermostat temperature, denoted as $T_{R T U_{\#}}$, is marked with a blue solid line while each corresponding zone temperature is marked with a red dashed line in Fig. 13a. The thermostat temperatures are associated with the circles in Fig. 11a and the zone temperatures are volume-averaged temperature ${ }^{5}$ over the zones shown in Fig. 11b.

The $T_{R T U_{2}}$ and $T_{R T U_{4}}$ histories in Fig. 13a show there are significant gaps between the thermostat temperatures and the wine bar zone air temperature. Although the zone air temperature is several degrees higher than the set-point, RTU2 is rarely used as shown in Fig. 13b. Furthermore the short cycling of RTU4 indicates that RTU4 also runs partially regardless of the higher zone air temperature. This is because thermostats 2 and 4 are highly affected by the operation of RTU1 (see Fig. 11a for their sensor locations) and the correlation between the ON/OFF stage profile of RTU1 and $T_{R T U_{2}}, T_{R T U_{4}}$ can be checked in Fig. 13a and 13b. Furthermore, as pointed out, the net flow from the wine bar to the dining area due to the supply and return vent system (see Fig. 11b) means that RTU1 can not meet the wine bar zonal load. Zone2 air temperature deviates up to $3^{\circ} \mathrm{C}$ from the set-point during the entire simulation period. Furthermore zone1 temperature is also around $1^{\circ} \mathrm{C}$ higher than $T_{R T U_{1}}$ as shown in Fig. 13a. This is attributed to the non-uniform heat gain injected into the zone not into the thermostat location.

The level of comfort violation and the control behaviors would be different depending on the conditions of weather and load. However the reduced-order indoor air coupled model allows to compare the building performances before and after the retrofit of the sensor locations under the same conditions.

Due to the poor location of thermostats 1,2 and 4, they were relocated from the arrowed circles to the arrowed triangles in Fig. 11a. Fig. 14a and 14b show the thermostat temperature profiles, the average zone air temperatures and $\mathrm{ON} / \mathrm{OFF}$ compressor stages after retrofitting the sensor locations. As expected, each temperature is very well regulated. It is of interest to compare the change of run-time fraction (RTF), which is defined as the fraction of the

\footnotetext{
${ }^{5}$ The volumes for averaging are as follows: $69 \mathrm{~m}^{3}$ for the dining area in yellow in Fig. 16 and $23 \mathrm{~m}^{3}$ volume for the wine bar area that surrounds the wine bar.
} 
Table 3: RTU run-time fractions comparisons before and after the sensor location retrofit (one month simulation period)

\begin{tabular}{|c|c|c|c|c|c|}
\hline & \multicolumn{2}{|c|}{ RTU1 } & \multirow{2}{*}{ RTU2 } & \multirow{2}{*}{ RTU3 } & RTU4 \\
\cline { 2 - 5 } & Stage1 & Stage2 & & & \\
\hline \hline Before & 0.61 & 0.33 & 0.02 & 0.42 & 0.18 \\
\hline After & 0.61 & 0.37 & 0.35 & 0.28 & 0.24 \\
\hline
\end{tabular}

Table 4: Predicted HVAC energy consumption before and after retrofitting thermostat locations (one month simulation period)

\begin{tabular}{|c|l|c|}
\hline & $\begin{array}{l}\text { Daily averaged energy } \\
\text { consumption [kWh/day] }\end{array}$ & Cost increase [\%] \\
\hline \hline Before & 253.96 & - \\
\hline After & 275.16 & 8.35 \\
\hline
\end{tabular}

ON period to total period, and the HVAC energy consumption. They are summarized in Table 3 and 4 . The RTF metric provides information on how each RTU is utilized for the one month operating period and demonstrates clearly how the RTU2 and RTU4 are more utilized to regulate the wine bar zone air temperature. Due to the higher utilization of RTU1, 2 and 4 , about $8 \%$ more HVAC energy is required (see Table 4).

\subsubsection{Further Discussions}

Our main motivation for developing the integrated modeling tool was for use as a virtual testbed for evaluating advanced controls for buildings that use multiple RTUs to serve a large open space. For example, we have developed an algorithm for optimal coordination of multiple RTUs that was fully evaluated using this virtual testbed prior to implementation testing at a field site. No other practical tools exist that could be employed for this purpose.

As demonstrated in the case study, the tool could also be useful for evaluating optimal placement of thermostats. However, it would be a significant effort to build a model for each specific case study that might come along. Our vision is to develop a tool chain that would automatically generate an integrated reduced-order model from a physical description of a building and its HVAC system that could then be used for advanced control and thermostat placement

analysis. Furthermore, the tool chain could be used to develop a range of different prototypical building models that could enable control and thermostat placement analysis for a wide range of situations encountered in the field. 


\section{CONCLUSION}

This paper presented a unique approach for simulating the dynamics of indoor environments and building envelopes using a reduced-order coupled CFD model. The model is particularly appropriate for studying building control performance in small commercial buildings that utilize rooftop units (RTUs) in open spaces. The approach enables investigation of spatial variations of zone air temperature with low computation cost of about one second per one day simulation period. The enhanced model was shown to accurately predict RTU return air temperature responses in comparison to measurements for a typical sitdown restaurant that employs four rooftop units. Although this approach requires a detailed CFD model to generate a reduced order indoor air model, it is envisioned that a library of models could be created for typical small commercial buildings that have similar floor plans (e.g. big box retail, small sit-down restaurants).

An application of the reduced-order CFD coupled model, a retrofit analysis with respect to the change of the thermostat locations on both comfort (temperatures) and energy consumption, which can not be investigated by any other traditional building simulation tools, was performed. The study was feasible due to the model capability to capture spatial variations of thermal comfort and its tractable computational requirements. For this case study, the computation time of the complete model, HVAC, controller and the CFD coupled model, was about $20 \mathrm{sec} /$ day. The results indicate that comfort could be significantly improved through relocation of the thermostats but at some the expense of some additional energy usage.

\section{ACKNOWLEDGMENT}

This work was supported by the Department of Energy through the Consortium for Building Energy Innovation (CBEI), under contract DE-EE0004261.

\section{APPENDIX}

\subsection{Development of a Thermal Building Envelope Model}

Kim and Braun [31] derived a general formula for converting the complex thermal network of a multi-zone building into a linear time invariant statespace representation. Although the majority of the derivations in this section are the same as the paper, the formulation is slightly modified in order to connect to a reduced-order indoor air model as described in this section. The net radiosity method for long-wave interaction is adopted to construct a general form which includes long-wave interaction among surfaces, conduction through walls, distributed thermal capacitance within walls. After developing the formula, the LTI model is used to couple a reduced-order CFD model. For a complete list of nomenclature, please refer to [31]. 


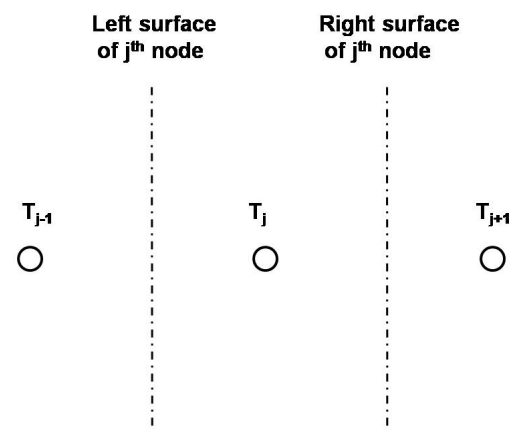

Figure 15: Notation for conduction through walls

\subsubsection{Conduction through Walls}

A finite volume formulation is used to describe the heat conduction through walls and is depicted in Fig. 15. For any $j^{\text {th }}$ node in a wall except the first and last nodes, an energy balance leads to

$$
\rho_{j}^{i} C_{j}^{i} w_{j}^{i} \frac{d T_{j}^{i}}{d t}=\left.h_{c d}^{L}\right|_{j} ^{i} T_{j-1}^{i}-\left(\left.h_{c d}^{L}\right|_{j} ^{i}+\left.h_{c d}^{R}\right|_{j} ^{i}\right) T_{j}^{i}+\left.h_{c d}^{R}\right|_{j} ^{i} T_{j+1}^{i}+q_{g e n, j}^{i}
$$

where $\left.h_{c d}^{L}\right|_{j} ^{i}=\left.k^{L}\right|_{j} ^{i} /\left.w^{L}\right|_{j} ^{i} .\left.k^{L}\right|_{j} ^{i}$ is thermal conductivity at left surface of the $j^{\text {th }}$ node in $i^{t h}$ wall, $\left.w^{L}\right|_{j} ^{i}$ is distance from the $(j-1)^{t h}$ node to the $j^{t h}$ node in $i^{\text {th }}$ wall, and $q_{g e n, j}^{i}$ is an energy source $\left(\mathrm{W} / \mathrm{m}^{2}\right)$ inside the $j^{\text {th }}$ finite control volume that belongs to the $i^{\text {th }}$ wall. By using the following matrix notation,

$$
\begin{aligned}
\vec{q}_{j} & =\left[\begin{array}{c}
q_{g e n, j}^{1} \\
q_{g e n, j}^{2} \\
\vdots \\
q_{g e n, j}^{N_{w}}
\end{array}\right], \vec{T}_{j}=\left[\begin{array}{c}
T_{j}^{1} \\
T_{j}^{2} \\
\vdots \\
T_{j}^{N_{w}}
\end{array}\right], \tilde{C}_{j}=\left[\begin{array}{cccc}
C_{j}^{1} & 0 & \ldots & 0 \\
0 & C_{j}^{2} & & 0 \\
\vdots & & \ddots & \vdots \\
0 & 0 & \ldots & C_{j}^{N_{w}}
\end{array}\right], \\
\tilde{H}_{j} & =\left[\begin{array}{cccc}
\left.h_{c d}\right|_{j} ^{1} & 0 & \ldots & 0 \\
0 & \left.h_{c d}\right|_{j} ^{2} & & 0 \\
\vdots & & \ddots & \vdots \\
0 & 0 & \ldots & \left.h_{c d}\right|_{j} ^{N_{w}}
\end{array}\right] .
\end{aligned}
$$

Eqn. 10 can be expressed in the following matrix form:

$$
\tilde{C}_{j} \frac{d \vec{T}_{j}}{d t}=\tilde{H}_{c d, j}^{L} \vec{T}_{j-1}-\left(\tilde{H}_{c d, j}^{L}+\tilde{H}_{c d, j}^{R}\right) \vec{T}_{j}+\tilde{H}_{c d, j}^{R} \vec{T}_{j+1}+\vec{q}_{j},
$$

where $N_{w}$ represents the number of walls in a zone (or a room). Note that $\vec{T}_{j}$ is a group of temperatures at all the wall nodes belonging to a zone. 


\subsubsection{Heat Balance at Outside Surface}

For any $i^{\text {th }}$ outside wall (connected to the external ambient) belonging to a zone, the heat balance equation at the surface is

$$
\rho_{1}^{i} C_{1}^{i} w_{1}^{i} \frac{d T_{1}^{i}}{d t}=h_{c v, e x}^{i}\left(T_{a}-T_{1}^{i}\right)+\left.h_{c d}^{R}\right|_{1} ^{i}\left(T_{2}^{i}-T_{1}^{i}\right)+\alpha_{1}^{i} q_{S W}^{i}+q_{L W}^{i},
$$

where $T_{1}^{i}$ represents the wall temperature of the first node which is set to be an outside surface of the wall. $q_{S W}^{i}$ and $q_{L W}^{i}$ represent short wavelength solar irradiation and net long wavelength radiation exchange with the environment, respectively. $h_{c v, e x}$ is a convective heat transfer coefficient at the outside surface of a wall and $T_{a}$ is outdoor air temperature, respectively. With the assumptions that the outside surface is gray and diffuse and the air is a non-participating radiation media, the net long wavelength interactions with the environment can be expressed as

$$
q_{L W}^{i}=\sigma \epsilon_{1}^{i} F_{s k y}^{i}\left(T_{s k y}^{4}-T_{1}^{i^{4}}\right)+\sigma \epsilon_{1}^{i} F_{g r d}^{i}\left(T_{g r d}^{4}-T_{1}^{i^{4}}\right),
$$

where $\sigma, \epsilon$ and $F$ are Stefan-Boltzmann constant, emittance and view factor, respectively. Using a simple linear approximation of the long-wave heat exchange term [40, ch. 10] gives

$$
\rho_{1}^{i} C_{1}^{i} w_{1}^{i} \frac{d T_{1}^{i}}{d t}=-\left(h_{c v, e x}^{i}+\left.h_{c d}^{R}\right|_{1} ^{i}+h_{r a d, e x}^{i}\right) T_{1}^{i}+h_{c v, e x}^{i} T_{2}^{i}+q_{1}^{i},
$$

where $h_{\text {rad,ex }}^{i}=4 \sigma \epsilon_{1}^{i}\left(F_{s k y}^{i} \bar{T}_{s k y}^{3}+F_{g r d}^{i} \bar{T}_{g r d}^{3}\right), \bar{T}_{s k y}=\left(T_{s k y}+T_{1}\right) / 2, \bar{T}_{g r d}=\left(T_{\text {grd }}+\right.$ $\left.T_{1}\right) / 2$ and $q_{1}^{i}=h_{c v, e x}^{i} T_{a}+4 \sigma \epsilon_{1}^{i}\left(F_{s k y}^{i} \bar{T}_{s k y}^{3} T_{s k y}+F_{g r d}^{i} \bar{T}_{g r d}^{3} T_{g r d}\right)+\alpha_{1}^{i} q_{S W}^{i}$. The mean temperatures for long-wave exchange between the surface and sky and surface and ground are assumed to be the same and constant for all outside surfaces for the purpose of linearization. TMY3 data and a nominal zone air temperature were used to determine the mean temperatures. Eqn. 14 can be generalized and written in compact matrix form as

$$
\tilde{C}_{1} \frac{d \vec{T}_{1}}{d t}=-\left(\tilde{H}_{c d, 1}^{R}+\tilde{H}_{r a d, e x}+\tilde{H}_{c v, e x}\right) \vec{T}_{1}+\tilde{H}_{c d, 1}^{R} \vec{T}_{2}+\vec{q}_{1}
$$

where the $i^{\text {th }}$ component of $\vec{q}_{1}$ is $q_{1}^{i}$.

\subsubsection{Heat Balance at Inside Surface}

For the $i^{t h}$ wall, the energy balance equation for the inside surface is

$$
\rho_{n}^{i} C_{n}^{i} w_{n}^{i} \frac{d T_{n}^{i}}{d t}=\left.h_{c d}^{L}\right|_{n} ^{i}\left(T_{n-1}^{i}-T_{n}^{i}\right)-q_{n e t, r a d}^{i}+q_{c v, i n}^{i},
$$

where $q_{n e t, r a d}^{i}$ is net radiative flux out of the inside wall and $q_{c v, \text { in }}^{i}$ is the net heat flux from zone air which allows the CFD coupling. The radiosity method is utilized to express the net flux assuming the walls are gray, diffuse and opaque. The same linearization method used in Eqn. 13 is employed leading to

$$
\vec{q}_{n e t, r a d}=\tilde{A}^{-1}\left[\tilde{B} \vec{T}_{n}-\vec{h}_{o}\right],
$$


where $\tilde{A}_{i j}=\frac{\delta_{i j}}{\epsilon_{j}}-\frac{\rho_{j}}{\epsilon_{j}} F_{i j}, \tilde{B}_{i j}=4 \sigma\left(\delta_{i j}-F_{i j}\right) \bar{T}^{3} . F_{i j}$ is view factor from $i$ to $j$ surface, $\delta$ is the Kronecker delta, $\rho$ is reflectance and $\vec{h}_{o}$ is external radiative source. A similar formulation for the treatment of long-wave interactions is shown in [41]. In Eqn. 17, radiosity does not appear explicitly, which is convenient for building simulation. Since the $i^{t h}$ component of $\vec{h}_{o}$ represents an external radiative source acting on the $i^{t h}$ surface, the effects of internal sources and transmitted solar energy though windows are treated in a consistent manner. For any shaped room, the net radiative flux can be explicitly calculated as a function of surface temperatures if the view factors and the external radiative sources are known. By letting $H_{\text {rad,in }}^{\sim} \equiv \tilde{A}^{-1} \tilde{B}$ and $\overrightarrow{q_{n}} \equiv \tilde{A}^{-1} \vec{h}_{o}$,

$$
\tilde{C}_{n} \frac{d \vec{T}_{n}}{d t}=\tilde{H}_{c d, n}^{L} \vec{T}_{n-1}-\left(\tilde{H}_{c d, n}^{L}+\tilde{H}_{r a d, i n}\right) \vec{T}_{n}+\vec{q}_{n}+\vec{q}_{c v, i n}
$$

\subsubsection{LTI Building Envelope Model}

Gathering the system of equations that represent heat balance equations for external to internal wall elements, i.e. Eqn. 11, 15 and 18,

$$
\begin{aligned}
{\left[\begin{array}{cccc}
\tilde{C}_{1} & 0 & \cdots & 0 \\
0 & \tilde{C}_{2} & & 0 \\
\vdots & & \ddots & \vdots \\
0 & 0 & \vdots & \tilde{C}_{n}
\end{array}\right]\left[\begin{array}{c}
\dot{\vec{T}}_{1} \\
\overrightarrow{\vec{T}}_{2} \\
\vdots \\
\vdots \\
\vec{T}_{n}
\end{array}\right]=} & {\left[\begin{array}{cccc}
-\tilde{H}_{1} & \tilde{H}_{c d, 1}^{R} & \ldots & 0 \\
\tilde{H}_{c d, 2}^{L} & -\left(\tilde{H}_{c d, 2}^{L}+\tilde{H}_{c d, 2}^{R}\right) & \tilde{H}_{c d, 2}^{R} & 0 \\
\vdots & & \ddots & \vdots \\
0 & 0 & \tilde{H}_{c d, n}^{L} & -\tilde{H}_{n}
\end{array}\right]\left[\begin{array}{c}
\vec{T}_{1} \\
\vec{T}_{2} \\
\vdots \\
\vec{T}_{n}
\end{array}\right] } \\
& +\left[\begin{array}{c}
\vec{q}_{1} \\
\vec{q}_{2} \\
\cdots \\
\vec{q}_{n}
\end{array}\right]+\left[\begin{array}{c}
0 \\
0 \\
\vdots \\
\vec{q}_{c v, i n}
\end{array}\right]
\end{aligned}
$$

where $\tilde{H}_{1}=\tilde{H}_{c d, 1}^{R}+\tilde{H}_{r a d, e x}$ and $\tilde{H}_{n}=\tilde{H}_{c d, n}^{L}+\tilde{H}_{r a d, i n}$.

The terms $\vec{q}_{j}$, where $j$ is from 2 to $n-1$, vanish if there are no heat flux sources inside the wall such as embedded radiant heating or cooling. Note that $\vec{q}_{1}$ contains weather information, e.g. outdoor air and solar irradiations, and $\vec{q}_{n}$ admits a radiative heat source such as lightening. $\vec{T}_{n}$ and $\vec{q}_{c v, i n}$ are the coupled parameters, $y_{1}$ and $v_{1}$ respectively in Eqn. 3. Based on Eqn. 19, one can easily get a building envelope system matrices $\left(A_{1}, F_{1}, B_{1}, G_{1}\right)$ in Eqn. 3. For the restaurant case study, all parameters, such as wall thickness, thermal conductivity and material density, were obtained or estimated from the construction drawings for the building. 


\subsection{Development of LTI Reduced-Order Indoor CFD Model}

\subsubsection{Standard Model}

Energy exchange between a bounding surface and the indoor-air is typically modeled in terms of a convective film coefficient as

$$
q_{j}(t)=h_{j}\left(T_{j}(t)-T_{\text {zone }}(t)\right),
$$

where $q_{j}(t), T_{j}(t)$ are the instantaneous flux $\left(\mathrm{w} / \mathrm{m}^{2}\right)$ and the temperature of the $j^{\text {th }}$ wall surface, respectively and $h_{j}$ is a film coefficient. The latter may be based on experimental results and depends on surface finish and orientation. Evolution of a fully-mixed zone temperature $\left(T_{z}\right)$ is commonly modeled as

$$
\mathcal{C}_{\mathrm{z}} \frac{\mathrm{d} T_{\mathrm{z}}(t)}{\mathrm{d} t}=\sum_{j=1}^{m} q_{j}(t)+S_{\mathrm{z}}(t),
$$

where $\mathcal{C}_{\mathrm{z}}$ is the thermal capacity of the well-mixed air $\left(\mathrm{J} /{ }^{\circ} \mathrm{K}\right)$, there are $m$ internal wall surfaces, and $S_{\mathrm{z}}(t)$ represents source terms in the zone.

If we identify system inputs $(u)$ with the segment temperatures $\left(T_{\imath}\right)$, and the outputs $(y)$ with the segment heat fluxes $\left(q_{j}\right)$, then the standard system (Eqn. 20, 21) is a linear time-invariant (LTI) system and in the usual first-order system form $(A, B, C, D)$ we can identify

$$
\begin{aligned}
& C=\left[\begin{array}{c}
h_{1} \\
h_{2} \\
\vdots \\
h_{m}
\end{array}\right], \quad D=\left[\begin{array}{ccccc}
h_{1} & 0 & 0 & \ldots & 0 \\
0 & h_{2} & 0 & \ldots & 0 \\
0 & 0 & \ddots & \vdots & \vdots \\
\vdots & \vdots & & \ddots & \vdots \\
0 & 0 & 0 & 0 & h_{m}
\end{array}\right] \\
& A=\frac{-\sum_{j=1}^{m} h_{j}}{\mathcal{C}_{\mathrm{z}}} \text { (a scalar), and } B=\frac{C^{T}}{\mathcal{C}_{\mathrm{z}}} .
\end{aligned}
$$

Note that Eqn. 22 does not account for the $(m+1)^{\text {st }}$ input $\left(S_{z}\right)$.

The Standard Model transfer function from the inputs (the internal surface temperatures $(T)$ ) to the outputs (the segment loads $(Q)$ ) is given by

$$
H^{\mathrm{std}}(s)=\frac{-C C^{T}}{\left(\mathcal{C}_{\mathrm{z}} s+\sum h_{j}\right)}+D,
$$

where the arrays $C, D$ depend on the wall-segment convective parameters $h_{j}$ as given in display Eqn. 22. Note that the transfer function $H^{\text {std }}$ is not strictly proper $(D \neq 0)$.

\subsection{2. $C F D$-based model}

Our indoor-air model is realized as a linear-time-invariant (LTI) system that approximates specific input-output behavior observed in computational fluid dynamics (CFD) simulations. A grid for our CFD simulation is shown in Fig. 16. 


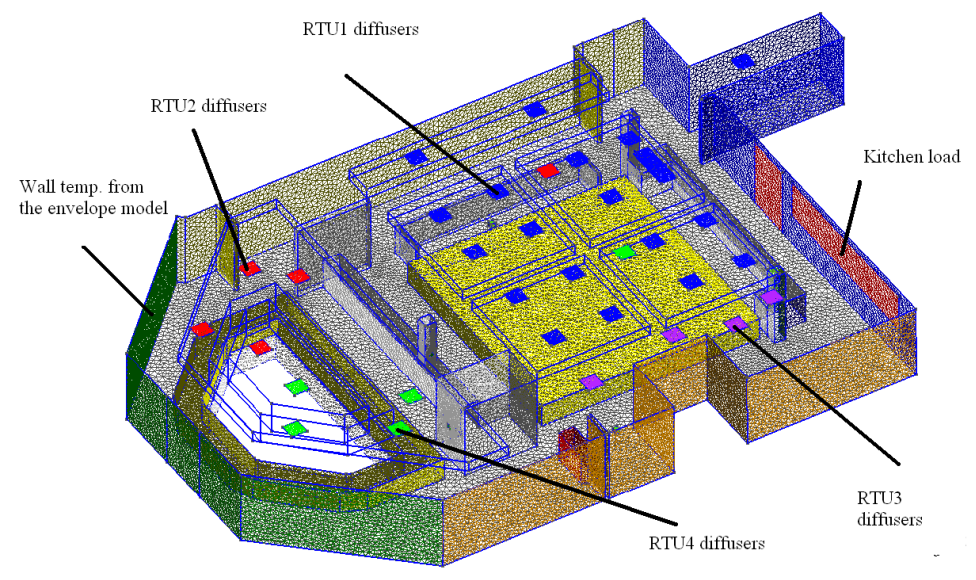

Figure 16: CFD grid and $\mathrm{BC}$ for a demonstration site

The building envelope model was coupled to a Standard Model for the indoor-air and subjected to fixed loads. The resulting steady surface temperatures were enforced as Dirichlet boundary conditions and the CFD simulation was run to a nominal steady-state. With a steady flow solution in hand, we then subjected the CFD model to a step-like change in each of the surface temperatures $\left(+5^{\circ} \mathrm{C}\right)$. In addition to the surface temperatures, we subjected the CFD model to perturbations in the supply air mass-rate and temperature, and to a change in occupancy load(s). In addition to the required surface fluxes, we recorded response in return air temperature, water-vapor and air quality metrics in various occupied zones. In all, 43 quantities were recorded.

ANSYS FLUENT 14.5 was used for the CFD calculations. The CFD mesh consisted of $311 \mathrm{~K}$ nodes and $1.68 \mathrm{M}$ tetrahedral cells. A single case with one level of mesh refinement was run with little observed change in the output quantities of interest.

FLUENT's implementation of the Spaulding-Patankar SIMPLE scheme was used for spatial discretization including: least-squares cell-based gradients, pressure via PRESTO, all flux calculations are 2nd-order upwind. The standard $\kappa-\epsilon$ turbulence model with standard wall functions was used, with an observed average $y^{+}$of about 38. Time discretization was first order implicit with a limit of 100 iterations per time step. Time steps were mostly 4 seconds and convergence was achieved within one or two iterations for all runs with recorded data.

On the inside of the exterior walls, ceiling and floor, specified temperatures were applied. We computed average heat fluxes for the bounding surfaces and generated data for a time-varying Dirichlet-to-Neumann map. Interior walls 
were zero-heat flux, and all solid surfaces were no-slip. Supply vents were modeled as 'velocity-inlets' with the velocity distribution a flatten parabola. Outflow (return vents) were 'pressure outlet' with specified hydrostatic pressure below the (floor-based) reference. No internal radiation was included in the CFD calculations. This is because radiative interactions are treated in the envelope side (see previous section) and is based on the fact that air is a radiatively non-participating media.

\subsubsection{Fitting the Data}

For each input $\left(u_{j}\right)$ we hypothesize an LTI model of the form

$$
\begin{aligned}
& \dot{x}_{j}(t)=-\frac{1}{\tau_{j}} x_{j}(t)+u_{j}(t) \\
& y^{j}(t)=C^{j} x_{j}(t)+D^{j} u_{j}(t),
\end{aligned}
$$

where $x_{j}$ is a scalar state, and the column matrices $C^{j}, D^{j} \in \mathbb{R}^{43}$. Based on the Standard Model (Eqn. 20) we have $D_{k}^{j}=0, k \neq j$ and $D_{j}^{j}=h_{j}$, the film coefficient for the $j^{\text {th }}$ surface.

The building envelope model exhibits more than ten eigenvalues (time constants) that are slower than 300 seconds, whereas the indoor air time constants for perturbations in the supply air or the occupant loads are of the order of 300 seconds. For this reason we insist that the asymptotic response of the model exactly match the data. For a unit-step input this implies that

$$
C^{j}=\frac{y_{\mathrm{ss}}^{j}-D^{j}}{\tau_{j}} .
$$

Thus, under the constraint that the steady-state output of the model exactly match the data, we have a single free-parameter $\left(v i z, \tau_{j}\right)$. This single parameter is found as a solution of a least-squares minimization problem;

$$
\min _{\tau} J(\tau) \triangleq \sum_{k}\left\|y_{\text {model }}\left(t_{k} ; \tau\right)-y_{\text {data }}\left(t_{k}\right)\right\|^{2} .
$$

This minimization was done using MATLAB's fminsearch procedure. For the case of bounding surface temperature inputs the cost function also depends on the $h$ parameter that is, we minimize $J(\tau, h)$.

\section{Reference}

[1] D. Crawley, L. Lawrie, C. Pedersen, F. Winkelmann, Energy plus: energy simulation program, ASHRAE journal 42 (4) (2000) 49-56, URL http: //apps1. eere.energy.gov/buildings/energyplus/.

[2] S. Klein, W. Beckman, J. Mitchell, J. Duffie, N. Duffie, T. Freeman, J. Mitchell, J. Braun, B. Evans, J. Kummer, et al., TRNSYS 16-A TRaNsient system simulation program, user manual, Solar Energy Laboratory. Madison: University of Wisconsin-Madison . 
[3] J. Srebric, Q. Chen, L. Glicksman, A coupled airflow and energy simulation program for indoor thermal environmental studies, Tech. Rep., Massachusetts Inst. of Tech., Cambridge, MA (US), URL http://www. osti. gov/energycitations/product.biblio.jsp?osti_id=20104808, 2000.

[4] Z. Zhai, Q. Y. Chen, Solution characters of iterative coupling between energy simulation and CFD programs, Energy and Buildings 35 (5) (2003) 493-505.

[5] J. Srebric, J. Yuan, A. Novoselac, On-site experimental validation of a coupled multizone and CFD model for building contaminant transport simulations, ASHRAE Transactions 114 (1).

[6] Z. Zhai, Q. Chen, Performance of coupled building energy and CFD simulations, Energy and buildings 37 (4) (2005) 333-344, URL http: //www.sciencedirect.com/science/article/pii/S0378778804002130.

[7] M. Bartak, I. Beausoleil-Morrison, J. Clarke, J. Denev, F. Drkal, M. Lain, I. Macdonald, A. Melikov, Z. Popiolek, P. Stankov, Integrating CFD and building simulation, Building and Environment 37 (8) (2002) 865-871, URL http://www.sciencedirect.com/science/article/pii/ S0360132302000458.

[8] E. Dascalaki, M. Santamouris, M. Bruant, C. Balaras, A. Bossaer, D. Ducarme, P. Wouters, Modeling large openings with COMIS, Energy and Buildings 30 (1) (1999) 105-115.

[9] G. N. Walton, W. S. Dols, CONTAM 2.4 user guide and program documentation, National Institute of Standards and Technology, NISTIR 7251.

[10] H. Boyer, A. P. Lauret, L. Adelard, T. A. Mara, Building ventilation: a pressure airflow model computer generation and elements of validation, Energy and Buildings 29 (3) (1999) 283-292.

[11] H. E. Feustel, J. Dieris, A survey of airflow models for multizone structures, Energy and buildings 18 (2) (1992) 79-100.

[12] J. Axley, Multizone airflow modeling in buildings: History and theory, HVAC\&R Research 13 (6) (2007) 907-928, URL http://www. tandfonline.com/doi/abs/10.1080/10789669.2007.10391462.

[13] E. Djunaedy, J. Hensen, M. Loomans, External coupling between CFD and energy simulation: implementation and validation, ASHRAE Transactions 111 (1) (2005) 612-624.

[14] Y. Fan, K. Ito, Energy consumption analysis intended for real office space with energy recovery ventilator by integrating BES and CFD approaches, Building and Environment 52 (2012) 57-67. 
[15] Z. Du, P. Xu, X. Jin, Q. Liu, Temperature sensor placement optimization for VAV control using CFD-BES co-simulation strategy, Building and Environment 85 (2015) 104-113.

[16] W. Zuo, Q. Chen, Fast and informative flow simulations in a building by using fast fluid dynamics model on graphics processing unit, Building and Environment 45 (3) (2010) 747-757.

[17] W. Zuo, M. Wetter, D. Li, M. Jin, W. Tian, Q. Chen, Coupled simulation of indoor environment, HVAC and control system by using fast fluid dynamics and the Modelica buildings library .

[18] M. Wetter, Modelica-based modelling and simulation to support research and development in building energy and control systems, Journal of Building Performance Simulation 2 (2) (2009) 143-161, URL http://www. tandfonline.com/doi/abs/10.1080/19401490902818259.

[19] T. Zerihun Desta, K. Janssens, A. Van Brecht, J. Meyers, M. Baelmans, D. Berckmans, CFD for model-based controller development, Building and environment 39 (6) (2004) 621-633, URL http://www.sciencedirect. com/science/article/pii/S0360132304000149.

[20] T. Zerihun Desta, S. Van Buggenhout, A. Van Brecht, J. Meyers, J. Aerts, M. Baelmans, D. Berckmans, Modelling mass transfer phenomena and quantification of ventilation performance in a full scale installation, Building and environment 40 (12) (2005) 1583-1590, URL http: //www.sciencedirect.com/science/article/pii/S0360132304003816.

[21] A. Van Brecht, S. Quanten, T. Zerihundesta, S. Van Buggenhout, D. Berckmans, Control of the 3-D spatio-temporal distribution of air temperature, International Journal of Control 78 (2) (2005) 88-99, URL http: //www.tandfonline.com/doi/abs/10.1080/00207170500036118.

[22] K. Hiyama, S. Kato, Y. Ishida, Thermal simulation: Response factor analysis using three-dimensional CFD in the simulation of air conditioning control, in: Building Simulation, vol. 3, Springer, 195-203, 2010.

[23] Z. Zhai, Q. Chen, P. Haves, J. Klems, On approaches to couple energy simulation and computational fluid dynamics programs, Building and Environment 37 (8) (2002) 857-864, URL http://www.sciencedirect.com/ science/article/pii/S0360132302000549.

[24] A. Fluent, 12.0 Theory Guide, ANSYS, Inc., Canonsburg, PA 15317.

[25] L. Ljung, T. Glad, Modeling of dynamic systems, Prentice Hall, URL http: //www.citeulike.org/group/160/article/751215, 1994.

[26] L. Ljung, et al., Theory and practice of recursive identification . 
[27] P. Van Overschee, B. De Moor, A unifying theorem for three subspace system identification algorithms, Automatica 31 (12) (1995) 1853-1864, URL http://www.sciencedirect.com/science/article/ pii/0005109895000720.

[28] M. Green, D. J. Limebeer, Linear robust control, Courier Dover Publications, 2012.

[29] K. Zhou, J. Doyle, Essentials of robust control, vol. 104, Prentice Hall Upper Saddle River, NJ, 1998.

[30] B. Moore, Principal component analysis in linear systems: Controllability, observability, and model reduction, Automatic Control, IEEE Transactions on 26 (1) (1981) 17-32, URL http://ieeexplore.ieee.org/xpls/abs_ all. $\mathrm{jsp}$ ?arnumber $=1102568$.

[31] D. Kim, J. E. Braun, A general approach for generating reduced-order models for large multi-zone buildings, Journal of Building Performance Simulation (2015) 1-14URL http://www.tandfonline.com/doi/pdf/10. 1080/19401493.2014.977952.

[32] D. Kim, W. Zuo, J. E. Braun, M. Wetter, Comparisons of Building System Modeling Approaches for Control System Design, Proceedings of BS2013, 13th Conference of International Building Performance Simulation Association, Chambry, France .

[33] S. B. Ayed, J. T. Borggaard, E. M. Cliff, A Reduced Order Model of the Indoor-Air Environment for Energy Efficient Building Studies, 19th IFAC World Congress .

[34] D. Ripple, G. W. Burns, M. G. Scroger, Assessment of uncertainties of thermocouple calibrations at NIST, US Department of Commerce, National Institute of Standards and Technology, 1994.

[35] L. Ljung (Ed.), System Identification (2Nd Ed.): Theory for the User, Prentice Hall PTR, Upper Saddle River, NJ, USA, ISBN 0-13-656695-2, 1999.

[36] S. Skogestad, I. Postlethwaite, Multivariable feedback control: analysis and design, vol. 2, Wiley New York, 2007.

[37] V. Putta, D. Kim, J. Cai, J. Hu, J. Braun, Dynamic programming based approaches to optimal rooftop unit coordination, Science and Technology for the Built Environment Submitted.

[38] D. Kim, J. E. Braun, J. Cai, D. L. Fugate, Development and Experimental Demonstration of a Plug-and-Play Multiple RTU Coordination Control Algorithm for Small/Medium Commercial Buildings, in: Energy and Buildings, submitted, 2015. 
[39] E. Plus, Engineering Reference Manual, Building Technologies Program, US Department of Energy (DOE) .

[40] A. Faghri, Y. Zhang, J. R. Howell, Advanced heat and mass transfer, Global Digital Press, 2010.

[41] M. F. Modest, Radiative heat transfer, Academic press, 2003. 